\title{
Immunoproteome of Aspergillus fumigatus Using Sera of Patients with Invasive Aspergillosis
}

Emylli D. Virginio ${ }^{1}$, Paula H. Kubitschek-Barreira ${ }^{1}$, Marjorie Vieira Batista ${ }^{2,3}$, Marcelo R. Schirmer ${ }^{4}$, Eliana Abdelhay ${ }^{4}$, Maria A. Shikanai-Yasuda ${ }^{2,5}$ and

Leila M. Lopes-Bezerra ${ }^{1, *}$

1 Laboratory of Cellular Mycology and Proteomics, Biology Institute, University of Rio de Janeiro State (UERJ), Rio de Janeiro 20550-013, Brazil;

E-Mails: emylli.dias@gmail.com (E.D.V.); paulahkb@gmail.com (P.H.K.-B.)

2 Laboratory of Immunology (LIM 48), Clinics Hospital and Department of Infectious and Parasitic Diseases, Faculty of Medicine, University of São Paulo, São Paulo 05403-000, Brazil;

E-Mails: marjorie.mi@gmail.com (M.V.B.); shikanaiyasuda@gmail.com (M.A.S.-Y.)

3 Clinics of Infectious Diseases, Clinics Hospital, Faculty of Medicine, University of São Paulo, São Paulo 05403-900, Brazil

4 National Cancer Institute, Center for Bone Marrow Transplants, Rio de Janeiro 20230-130, Brazil; E-Mails: mschirmer@inca.gov.br(M.R.S.); eabdelhay@inca.gov.br (E.A.)

5 Department of Infectious and Parasitic Diseases, Faculty of Medicine, University of São Paulo, São Paulo 05403-000, Brazil

* Author to whom correspondence should be addressed; E-Mail: leila@uerj.br; Tel.: +55-21-2334-0835.

Received: 13 May 2014; in revised form: 24 July 2014 / Accepted: 8 August 2014 / Published: 20 August 2014

\begin{abstract}
Invasive aspergillosis is a life-threatening lung or systemic infection caused by the opportunistic mold Aspergillus fumigatus. The disease affects mainly immunocompromised hosts, and patients with hematological malignances or who have been submitted to stem cell transplantation are at high risk. Despite the current use of Platelia ${ }^{\mathrm{TM}}$ Aspergillus as a diagnostic test, the early diagnosis of invasive aspergillosis remains a major challenge in improving the prognosis of the disease. In this study, we used an immunoproteomic approach to identify proteins that could be putative candidates for the early diagnosis of invasive aspergillosis. Antigenic proteins expressed in the first steps of A. fumigatus germination occurring in a human host were revealed using 2-D Western immunoblots with the serum of patients who had previously been classified as probable and proven for
\end{abstract}


invasive aspergillosis. Forty antigenic proteins were identified using mass spectrometry (MS/MS). A BLAST analysis revealed that two of these proteins showed low homology with proteins of either the human host or etiological agents of other invasive fungal infections. To our knowledge, this is the first report describing specific antigenic proteins of $A$. fumigatus germlings that are recognized by sera of patients with confirmed invasive aspergillosis who were from two separate hospital units.

Keywords: immunoproteome; antigens; invasive aspergillosis; diagnosis

\section{Introduction}

Invasive aspergillosis is a life-threatening lung or systemic infection that primarily affects hematological patients under chemotherapy and hematopoietic stem cell transplant (HSCT) patients [1]. The infection is fatal in $30 \%-90 \%$ of the patients, including those given treatment [2]. The main etiological agent of invasive aspergillosis is the opportunistic mold Aspergillus fumigatus, which is responsible for $90 \%$ of aspergillus infections [3].

A confirmed diagnosis of invasive aspergillosis remains challenging and is frequently not achieved until necropsy. The isolation of aspergilli from cultures lacks sensitivity and, therefore, is ineffective for the diagnosis of invasive aspergillosis; blood cultures are rarely positive even in patients with confirmed invasive aspergillosis [4,5]. Moreover, the isolation of aspergilli in blood cultures or in sputum samples does not necessarily indicate the presence of the invasive disease. Positive results usually represent only colonization due the high colonization rate in immunocompromised patients; thus, false-positive results due environmental contamination are frequent [5,6].

The "gold standard" for the diagnosis of invasive aspergillosis remains histopathological examination or biopsy; however, this often requires invasive procedures to obtain tissue for the examination. In most cases, the aggressiveness of the underlying disease, as well as the toxic effects of the hematological therapies, make this type of examination impossible in critically ill patients $[3,7,8]$.

Currently, the routine techniques used for the diagnosis of invasive aspergillosis are computational tomography (CT) and the ELISA test for galactomannan (GM) (Platelia ${ }^{\mathrm{TM}}$ Aspergillus-BioRad, Hercules, CA, USA); these are considered along with microbiological findings and the clinical signs and symptoms of the patient $[9,10]$. The GM molecule is an immunodominant cell wall polysaccharide of Aspergillus and Penicillium species that is released during fungus growth [11,12]. Although it provides a fast serological result, the efficacy of the GM test remains controversial and varies depending on the clinic or health center, as previously reviewed by Xavier et al. [13]. False-positives have also been reported, for example, following treatment with a beta-lactam antibiotic; however, recent reports suggest that the new preparations of piperacillin-tazobactam do not test positive with galactomannan. Cross-reactions with fungi, such as Fusarium spp., Penicillium, Cladosporium and Histoplasma have also been reported [14-17]. The mean specificity of the test is $85 \%$ and the sensitivity varies from $29 \%$ to $100 \%[9,13]$.

The difficulties in reaching an early and precise diagnosis are also true for other invasive fungal infections. To define and classify the main invasive fungal infections in immunocompromised patients, 
the European Organization for Research and Treatment of Cancer/Invasive Fungal Infections Cooperative Group and the National Institute of Allergy and Infectious Diseases Mycoses Study Group (EORTC/MSG) Consensus Group created and revised the definitions for clinical and epidemiological research. According to the definitions, invasive fungal infections are classified as "proven", "probable", or "possible" $[5,18]$. Thus, there remains an urgent need to develop new diagnostic tools to prevent the onset of the disease.

The sequencing of the A. fumigatus genome and the advances in the proteomic field have made it feasible to study and identify putative candidates for the immunodiagnosis of invasive aspergillosis. Few antigens specific for allergic bronchopulmonary aspergillosis (ABPA), aspergilloma, and invasive aspergillosis are known and/or being evaluated for diagnosis [19]. Furthermore, some studies have already shown the potential of some proteins as biomarkers for the immunodiagnosis of invasive aspergillosis; however, none of these came to a clinical trial [20-24].

In this context, the aim of this study was to investigate the antigenic proteins revealed by patients' sera using cell wall extracts of $A$. fumigatus germlings in an attempt to find putative candidates for the diagnosis of invasive aspergillosis.

\section{Results and Discussion}

\subsection{Western Immunoblots and Antigenic Proteins Identified}

In recent decades, invasive fungal infections (IFI) have been considered the most important cause of morbidity and mortality in severely immunosuppressed patients. Although candidiasis remains the most frequent IFI in critically ill patients, aspergillosis and mucormycosis have also emerged as significant causes of morbidity and mortality. HSCT recipients and patients with prolonged neutropenia represent the main risk group for invasive aspergillosis [25]. In these patients, A. fumigatus is by far the most important etiological agent of invasive aspergillosis, especially in HSCT patients with acute leukemia (5\% to $25 \%$ ) and in some solid organ transplantation patients $[3,7,26]$.

As mentioned previously, the actual diagnostic methods lack specificity and sensitivity for the early diagnosis of invasive aspergillosis. In this context, many efforts have been undertaken to identify new molecular tools that could reduce this difficulty. Immunoproteomic-based antigen identification is a convenient tool that is widely used to indicate putative candidates for the molecular diagnosis of fungal infections, including invasive aspergillosis [23,24,27,28]. Germlings are cells in an early stage of growth, and surface proteins in this morphotype may play an essential role in the fungal-host interaction [29,30]. In addition, the cell surface location of these proteins makes germlings more easily recognized by the host immune system [12]. Thus, proteins present in the A. fumigatus germling cell wall can represent important putative antigenic markers for the early diagnostic of invasive aspergillosis.

In this study, the antigenic profile of cell surface proteins of A. fumigatus germlings $\left(\mathrm{GT}_{6} \mathrm{~h}\right)$ were identified through an immunoproteomic approach. The 2-DE profile of the $\mathrm{GT}_{6 \mathrm{~h}}$ extract, obtained as previously described [31], is shown in Figure 1. All antigenic proteins identified in this study, as well as their molecular mass, isoelectric points and functions, are listed in Table 1. The Western immunoblot analysis using the distinct pools of human sera, which were typed following 
the EORTC/MSG criteria as proven/Hospital 1; proven/Hospital 2 and probable, are shown in Figure $2 \mathrm{~A}-\mathrm{C}$; the correspondent antigenic proteins recognized by each pool of sera are listed in Table 2 .

As control, the $\mathrm{GT}_{6 \mathrm{~h}}$ extract was probed with sera from patients with underlying diseases similar to those found in the invasive aspergillosis proven patients. It is important to note that these patients did not receive antifungical therapy, did not develop any fungal disease, and survived for at least one month (data not shown). The immunoblot performed with the control sera revealed positive spots that corresponded to ten antigenic proteins (Table 2). Some of these proteins had already been described as A. fumigatus antigens in other studies using the sera of immunized rabbits, mice, and patients with a clinical suspicion of allergic bronchopulmonary aspergillosis [20,21,23,28,32], suggesting that they could be putative biomarkers for aspergillosis. However, our data suggest that these antigens can cross-react with the control pool of sera, indicating that they are unspecific for diagnostic purposes.

An important feature on diagnostic tests is their discriminate capacity among pathologies that can be clinically similar. Some studies demonstrate that the diagnosis of invasive aspergillosis can be confused with a range of other invasive fungal infections, such as paracoccidioidomycosis, fusariosis and mucormycosis [29-33]. This scenario emphasizes the need for more selective diagnostic methods for the diagnosis of invasive fungal infections, including invasive aspergillosis. In this context, we also tested a pool of sera from patients with other invasive mycoses (Figure 2D) including histoplasmosis, fusariosis, cryptococcosis and paracoccidioidomycosis. Positive spots correspondent to twenty-two proteins were revealed with this pool of sera (other mycoses) (Table 2). This cross-reactivity observed suggest that these proteins lack specificity for diagnostic purposes of invasive aspergillosis; these were not considered for further analysis.

Figure 1. Proteomic profile 2-DE of $\mathrm{TG}_{6 \mathrm{~h}}$ cell wall extract of $A$. fumigatus. Seventy-five $\mathrm{mg}$ of proteins were fractionated on $\mathrm{pH}$ 3-11 non-linear gradient 18-cm IPG strips followed by $12 \%$ homogenous 2 -D SDS PAGE. Proteins were visualized via silver staining. The identified antigenic spots are indicated using Arabic numbers or by Letters. $\mathrm{IEF}=$ Isoelectric Focusing. IPG $=$ Immobilized $\mathrm{pH}$ gradient. NL $=$ Non linear.

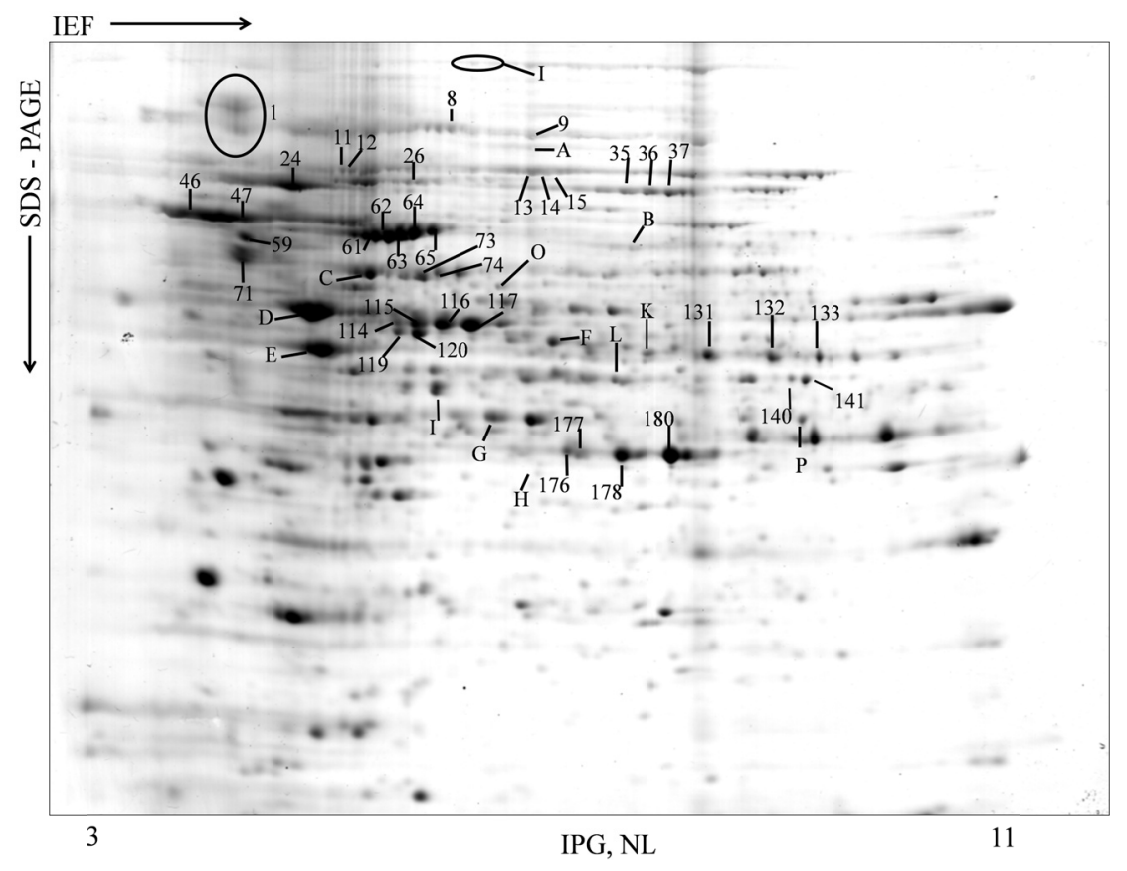


Table 1. All antigenic proteins identified via mass spectrometry (MS/MS) in the cell surface extracts of A. fumigatus.

\begin{tabular}{|c|c|c|c|c|c|c|c|c|c|c|c|}
\hline Spot & $\begin{array}{l}\text { Cov. } \\
(95 \%) \\
\end{array}$ & $\begin{array}{l}\text { Accession } \\
\text { Number }\end{array}$ & $\begin{array}{c}\text { EC } \\
\text { Number } \\
\end{array}$ & $\begin{array}{c}\text { Theorical } \\
M_{\mathrm{r}} / \mathbf{P I}\end{array}$ & Orf & Organism & Name & Function & Peptides & Conf. & Sequence \\
\hline 1 & 4.60 & Q4WC60 & 3.2 .1 .21 & $58.148 / 4.93$ & AFUA_8G05610 & A. fumigatus & $\begin{array}{c}\text { Probable } \\
\beta \text {-glucosidase btgE } \\
\end{array}$ & $\begin{array}{c}\text { Degradation of } \\
\text { cellulosic biomass }\end{array}$ & 2 & $\begin{array}{l}99 \\
99\end{array}$ & $\begin{array}{c}\text { EPGQFGVER } \\
\text { VYSTDCNSLEYIGEAAR }\end{array}$ \\
\hline 8 & 5.02 & Q4X1G3 & 6.3 .5 .5 & $129.214 / 5.87$ & AFUA_2G10070 & A. fumigatus & $\begin{array}{l}\text { Carbamoyl-phosphate } \\
\text { synthase, large subunit }\end{array}$ & $\begin{array}{l}\text { Nitrogen compound } \\
\text { metabolic process }\end{array}$ & 4 & $\begin{array}{l}99 \\
99 \\
99 \\
99\end{array}$ & $\begin{array}{c}\text { FAESVGYPVLVR } \\
\text { QIALLVGSTEDDVR } \\
\text { AAESVGYPIIVR } \\
\text { LADEVYYLPVTPEYVTHVIER }\end{array}$ \\
\hline $\begin{array}{l}11 \\
12 \\
13 \\
14 \\
15\end{array}$ & 8.94 & Q4X0G7 & - & $93.198 / 6.51$ & AFUA_2G13530 & A. fumigatus & $\begin{array}{c}\text { Translation elongation } \\
\text { factor EF-2 subunit, } \\
\text { putative }\end{array}$ & $\begin{array}{l}\text { Translation elongation } \\
\text { factor activity }\end{array}$ & 6 & $\begin{array}{l}99 \\
99 \\
99 \\
99 \\
99 \\
99\end{array}$ & $\begin{array}{c}\text { GHVYSEEQRPGTPLFNVK } \\
\text { ALGDVQVYPDR } \\
\text { AYLPVNESFGFNGDLR } \\
\text { DLEEDHAGVPLR } \\
\text { VNFTIEEIR } \\
\text { FSVSPVVQR }\end{array}$ \\
\hline $\begin{array}{l}13 \\
15\end{array}$ & 5.45 & B0XND2 & - & $81.445 / 5.74$ & AFUB_004530 & A. fumigatus & $\begin{array}{c}\text { Polyadenylate-binding } \\
\text { protein }\end{array}$ & RNA-binding & 3 & $\begin{array}{l}99 \\
98 \\
99\end{array}$ & $\begin{array}{c}\text { NIDQEVTDEEFR } \\
\text { NLTDDVDDEKLR } \\
\text { SLGYAYVNYNNTADGER }\end{array}$ \\
\hline
\end{tabular}


Table 1. Cont.

\begin{tabular}{|c|c|c|c|c|c|c|c|c|c|c|c|}
\hline Spot & $\begin{array}{c}\text { Cov. } \\
(95 \%) \\
\end{array}$ & $\begin{array}{c}\text { Accession } \\
\text { Number }\end{array}$ & $\begin{array}{c}\text { EC } \\
\text { Number }\end{array}$ & $\begin{array}{c}\text { Theorical } \\
M_{\mathrm{r}} / \mathbf{P I} \\
\end{array}$ & Orf & Organism & Name & Function & Peptides & Conf. & Sequence \\
\hline \multirow{13}{*}{24} & \multirow{5}{*}{9.66} & \multirow{5}{*}{ Q6MYM4 } & \multirow{5}{*}{ - } & \multirow{5}{*}{$80.04 / 5.08$} & \multirow{5}{*}{ AfA5C5.047 } & \multirow{5}{*}{ A. fumigatus } & \multirow{5}{*}{$\begin{array}{l}\text { Heat shock protein } \\
\text { Hsp88, putative }\end{array}$} & \multirow{5}{*}{ Response to stress } & \multirow{5}{*}{5} & 99 & FIAGPIVQR \\
\hline & & & & & & & & & & 99 & KNELESTIYELR \\
\hline & & & & & & & & & & 99 & LDLPGPEEKPR \\
\hline & & & & & & & & & & 99 & STPTLVGFGTR \\
\hline & & & & & & & & & & 99 & TLSFTLNQDEAIAR \\
\hline & \multirow{8}{*}{14.16} & \multirow{8}{*}{ P40292 } & \multirow{8}{*}{-} & \multirow{8}{*}{$80.64 / 4.94$} & \multirow{8}{*}{ AFUA_5G04170 } & \multirow{8}{*}{ A. fumigatus } & \multirow{8}{*}{$\begin{array}{l}\text { Heat shock protein } \\
\text { 90-Heat shock protein } \\
\text { hsp1 (Asp f 12) }\end{array}$} & & \multirow{8}{*}{8} & 99 & ADLINNLGTIAR \\
\hline & & & & & & & & Promotes maturation, & & 99 & GVVDSEDLPLNLSR \\
\hline & & & & & & & & structural maintenance and & & 99 & HFSVEGQLEFR \\
\hline & & & & & & & & pioper ieguialionit & & 99 & IILHLKDEQTDYLNESR \\
\hline & & & & & & & & D & & 99 & RAPFDLFETK \\
\hline & & & & & & & & 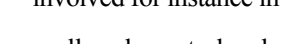 & & 99 & TGQFGWSANMER \\
\hline & & & & & & & & cell cycle control and & & 98 & LGIHEDAQNR \\
\hline & & & & & & & & signal transquetion & & 96 & DFELEETEEEKAER \\
\hline \multirow{4}{*}{26} & \multirow{4}{*}{7.83} & \multirow{4}{*}{ Q4WDH1 } & \multirow{4}{*}{ 6.3.4.13 } & \multirow{4}{*}{$86.42 / 5.31$} & \multirow{4}{*}{ AFUA_6G04730 } & \multirow{4}{*}{ A. fumigatus } & \multirow{4}{*}{$\begin{array}{c}\text { Bifunctional purine } \\
\text { biosynthetic protein } \\
\text { Ade1, putative }\end{array}$} & de novo IMP biosynthetic & \multirow{4}{*}{4} & 99 & EGEVVYQVGELKPR \\
\hline & & & & & & & & process, purine & & 99 & GLAHITGGGLVENVPR \\
\hline & & & & & & & & nucleobase & & 99 & HNIPTAAYQNFYEYEPAR \\
\hline & & & & & & & & biosynthetic process & & 99 & VIASTATASTLEEALR \\
\hline \multirow{6}{*}{$\begin{array}{l}35 \\
36 \\
37\end{array}$} & \multirow{6}{*}{8.77} & \multirow{6}{*}{ Q4WLN1 } & \multirow{6}{*}{ 4.2.1.3 } & & & & & & & 99 & FTGEYDAVPATAR \\
\hline & & & & & & & & & & 99 & HLGGLAIITR \\
\hline & & & & $05-0 \mathrm{~kg}$ & 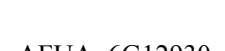 & 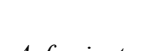 & Mitochondrial aconitate & Mitochondrial & 6 & 99 & LQRPLTYAEK \\
\hline & & & & $83.53 / 6.26$ & AFUA_6U12930 & A. fumlgatus & hydratase, putative & genome maintenance & 6 & 99 & QHIGDFAR \\
\hline & & & & & & & & & & 99 & SLFTVTPGSEQIR \\
\hline & & & & & & & & & & 99 & WVVIGDWNYGEGSSR \\
\hline
\end{tabular}


Table 1. Cont.

\begin{tabular}{|c|c|c|c|c|c|c|c|c|c|c|c|}
\hline Spot & $\begin{array}{l}\text { Cov. } \\
(95 \%)\end{array}$ & $\begin{array}{c}\text { Accession } \\
\text { Number }\end{array}$ & $\begin{array}{c}\text { EC } \\
\text { Number }\end{array}$ & $\begin{array}{c}\text { Theorical } \\
M_{\mathrm{r}} / \mathbf{P I} \\
\end{array}$ & Orf & Organism & Name & Function & Peptides & Conf. & Sequence \\
\hline \multirow{6}{*}{$\begin{array}{l}46 \\
47\end{array}$} & \multirow{6}{*}{12.66} & \multirow{6}{*}{ Q4WJ30 } & \multirow{6}{*}{-} & \multirow{6}{*}{$69.66 / 5.08$} & \multirow{6}{*}{ AFUA_1G07440 } & \multirow{6}{*}{ A. fumigatus } & \multirow{6}{*}{$\begin{array}{c}\text { Molecular } \\
\text { chaperone Hsp70 }\end{array}$} & \multirow{6}{*}{$\begin{array}{c}\text { ATP and nucleotide } \\
\text { binding; protein refolding }\end{array}$} & \multirow{6}{*}{6} & 99 & ATAGDTHLGGEDFDNR \\
\hline & & & & & & & & & & 99 & DAGLIAGLNVLR \\
\hline & & & & & & & & & & 99 & FELTGIPPAPR \\
\hline & & & & & & & & & & 99 & SSVHEIVLVGGSTR \\
\hline & & & & & & & & & & 99 & TTPSFVAFTDTER \\
\hline & & & & & & & & & & 96 & LVNHFVNEFKR \\
\hline \multirow{4}{*}{59} & \multirow{4}{*}{11.37} & \multirow{4}{*}{ Q4WMB7 } & \multirow{4}{*}{-} & \multirow{4}{*}{$53.56 / 4.58$} & \multirow{4}{*}{ AFUA_6G10470 } & \multirow{4}{*}{ A. fumigatus } & \multirow{4}{*}{ Zinc finger protein ZPR1 } & \multirow{4}{*}{$\begin{array}{l}\text { Cellular response to } \\
\text { starvation; regulation of } \\
\text { mitotic cell cycle }\end{array}$} & \multirow{4}{*}{4} & 99 & DIILESFECEHCGHR \\
\hline & & & & & & & & & & 99 & FTTVEGLLTEIR \\
\hline & & & & & & & & & & 99 & GESQLTTVEGVIQR \\
\hline & & & & & & & & & & 99 & YTLDVENEEDFQR \\
\hline \multirow{4}{*}{$\begin{array}{l}61 \\
62\end{array}$} & \multirow{4}{*}{7.98} & \multirow{4}{*}{ Q4WCM2 } & \multirow{4}{*}{-} & \multirow{4}{*}{$66.97 / 5.30$} & \multirow{4}{*}{ AFUA_8G03930 } & \multirow{4}{*}{ A. fumigatus } & \multirow{4}{*}{$\begin{array}{c}\text { Hsp70 chaperone (HscA), } \\
\text { putative }\end{array}$} & \multirow{4}{*}{$\begin{array}{l}\text { Protein refolding; } \\
\text { ATP-binding }\end{array}$} & \multirow{4}{*}{4} & 99 & AVITVPAYFNDNQR \\
\hline & & & & & & & & & & 99 & DAGAIAGLNVLR \\
\hline & & & & & & & & & & 99 & QQLESYISR \\
\hline & & & & & & & & & & 99 & SQVDEIVLVGGSTR \\
\hline \multirow{7}{*}{$\begin{array}{l}63 \\
64 \\
65\end{array}$} & \multirow{7}{*}{14.01} & & & & & & & & & 99 & DAGQIAGLNVLR \\
\hline & & & & & & & & & & 99 & IVQHTNGDAWVEAR \\
\hline & & & & & & & Mitochondrial Hsp70 & & & 99 & LLGNFQLVGIPPAHR \\
\hline & & Q4X1H5 & - & $74.46 / 6.02$ & AFUA_2G09960 & A. fumigatus & chaperone (Ssc70), & Protein refolding; protein & 7 & 99 & NAVVTVPAYFNDSQR \\
\hline & & & & & & & putative & targeting to mitocnondrion & & 99 & SQLESLVEPLINR \\
\hline & & & & & & & & & & 99 & TTPSVVAFAQDGER \\
\hline & & & & & & & & & & 98 & YSPSQIGGFILQK \\
\hline
\end{tabular}


Table 1. Cont.

\begin{tabular}{|c|c|c|c|c|c|c|c|c|c|c|c|}
\hline Spot & $\begin{array}{l}\text { Cov. } \\
(95 \%) \\
\end{array}$ & $\begin{array}{c}\text { Accession } \\
\text { Number }\end{array}$ & $\begin{array}{c}\text { EC } \\
\text { Number }\end{array}$ & $\begin{array}{c}\text { Theorical } \\
M_{\mathrm{r}} / \mathbf{P I} \\
\end{array}$ & Orf & Organism & Name & Function & Peptides & Conf. & Sequence \\
\hline \multirow{4}{*}{71} & 10.47 & A1D3E6 & - & $46.64 / 4.69$ & NFIA_016350 & A. fischerianus & $\begin{array}{c}\text { Protein phosphatase } 2 \mathrm{C} \\
\text { putative }\end{array}$ & $\begin{array}{c}\text { Protein } \\
\text { dephosphorylation }\end{array}$ & 3 & $\begin{array}{l}99 \\
99 \\
99\end{array}$ & $\begin{array}{c}\text { ISAAGGFVDFGR } \\
\text { NQFEETPDNYDLENDR } \\
\text { VANGDGPCAPPEYAEFR }\end{array}$ \\
\hline & 8.35 & Q4WU69 & - & $54.25 / 4.50$ & AFUA_5G07390 & A. fumigatus & $\begin{array}{c}\text { 60S ribosome biogenesis } \\
\text { protein Sqt1, putative }\end{array}$ & $\begin{array}{l}\text { Structural constituent } \\
\text { of ribosome }\end{array}$ & 3 & $\begin{array}{l}99 \\
99 \\
96\end{array}$ & $\begin{array}{c}\text { GEYVVTAGLDGR } \\
\text { VEFLQTNLAALASR } \\
\text { DERPVLPQSYESNPQPK }\end{array}$ \\
\hline & 8.98 & Q4WTN7 & - & $48.34 / 4.43$ & AFUA_5G05540 & A. fumigatus & $\begin{array}{l}\text { Nucleosome assembly } \\
\text { protein Nap1, putative }\end{array}$ & Nucleosome assembly & 3 & $\begin{array}{l}99 \\
99 \\
99\end{array}$ & $\begin{array}{l}\text { EESLDHATAASLFAR } \\
\text { SSGYIESLPAPVR } \\
\text { MEYLDRPGFR }\end{array}$ \\
\hline & 2.71 & Q4WH99 & 5.3.4.1 & $56.19 / 4.58$ & AFUA_2G06150 & A. fumigatus & $\begin{array}{c}\text { Protein disulfide } \\
\text { isomerase Pdi1, putative }\end{array}$ & $\begin{array}{c}\text { Cell redox } \\
\text { homeostasis; glycerol } \\
\text { ether metabolic process }\end{array}$ & 1 & 99 & AANDVFTSFAESQR \\
\hline \multirow[b]{2}{*}{$\begin{array}{l}73 \\
74\end{array}$} & 7.11 & Q4WXF1 & 5.4 .2 .1 & $57.45 / 5.44$ & AFUA_3G09290 & A. fumigatus & $\begin{array}{c}\text { Phosphoglycerate mutase, } \\
\text { 2,3-bisphosphoglycerate- } \\
\text { independent }\end{array}$ & $\begin{array}{c}\text { Glucose } \\
\text { catabolic process }\end{array}$ & 3 & $\begin{array}{l}99 \\
99 \\
99 \\
\end{array}$ & $\begin{array}{l}\text { VQDNDTLFFFNYR } \\
\text { EIGIGEIATVVGR } \\
\text { EITQLLGDYDR } \\
\end{array}$ \\
\hline & 15.26 & Q4WGP1 & 2.3 .1 .12 & $52.03 / 6.26$ & AFUA_7G05720 & A. fumigatus & $\begin{array}{l}\text { Pyruvate dehydrogenase } \\
\text { complex, dihydrolipoamide } \\
\text { acetyltransferase } \\
\text { component, putative }\end{array}$ & $\begin{array}{c}\text { Acetyl-CoA } \\
\text { biosynthetic process } \\
\text { from pyruvate }\end{array}$ & 5 & $\begin{array}{l}99 \\
99 \\
99 \\
99 \\
99\end{array}$ & $\begin{array}{c}\text { FTAVINPPQAAILAVGTTR } \\
\text { LQPSLDREPNISPAAK } \\
\text { NVHSLGLSSISNQIK } \\
\text { VPAVNSSWR } \\
\text { ENPHFFVSTTLSVTK }\end{array}$ \\
\hline $\begin{array}{l}114 \\
117\end{array}$ & 22.60 & Q96X30 & 4.2.1.11 & $47.31 / 5.39$ & AFUA_6G06770 & A. fumigatus & Enolase (Asp f 22) & $\begin{array}{c}\text { Glycolysis;regulation } \\
\text { of vacuole fusion, } \\
\text { non-autophagic }\end{array}$ & 6 & $\begin{array}{l}99 \\
99 \\
99 \\
99 \\
99 \\
99\end{array}$ & $\begin{array}{c}\text { AIVPSGASTGQHEAHELR } \\
\text { DSYADNWGVMVSHR } \\
\text { GNPTVEVDVVTETGLHR } \\
\text { GVPLYAHISDLAGTK } \\
\text { SGETEDVTIADIAVGLR } \\
\text { TSDFQIVGDDLTVTNPGR }\end{array}$ \\
\hline
\end{tabular}


Table 1. Cont.

\begin{tabular}{|c|c|c|c|c|c|c|c|c|c|c|c|}
\hline Spot & $\begin{array}{l}\text { Cov. } \\
(95 \%) \\
\end{array}$ & $\begin{array}{l}\text { Accession } \\
\text { Number }\end{array}$ & $\begin{array}{c}\text { EC } \\
\text { Number }\end{array}$ & $\begin{array}{c}\text { Theorical } \\
M_{\mathrm{r}} / \mathbf{P I} \\
\end{array}$ & Orf & Organism & Name & Function & Peptides & Conf. & Sequence \\
\hline \multirow{5}{*}{$\begin{array}{l}119, \\
120\end{array}$} & \multirow{5}{*}{12.75} & \multirow{5}{*}{ Q4WS30 } & \multirow{5}{*}{3.4 .24 .64} & \multirow{5}{*}{$53.27 / 5.90$} & \multirow{5}{*}{ AFUA_1G14200 } & \multirow{5}{*}{ A. fumigatus } & \multirow{5}{*}{$\begin{array}{c}\text { Mitochondrial processing } \\
\text { peptidase beta subunit, } \\
\text { putative }\end{array}$} & \multirow{5}{*}{$\begin{array}{c}\text { Metalloendopeptidase } \\
\text { activity }\end{array}$} & \multirow{5}{*}{5} & 99 & ASILLSLDGTTAVAEDIGR \\
\hline & & & & & & & & & & 99 & ITEKDVMDFANR \\
\hline & & & & & & & & & & 99 & LCYNVSAAEVER \\
\hline & & & & & & & & & & 99 & LNDLVHFALR \\
\hline & & & & & & & & & & 99 & TPEFIGSEIR \\
\hline 120 & 4.70 & Q5AZS8 & - & $49.75 / 9.88$ & AN6202.2 & A. nidulans & $\begin{array}{l}\text { RL3_NEUCR 60S } \\
\text { ribosomal protein L3 }\end{array}$ & $\begin{array}{c}\text { Structural constituent } \\
\text { of ribosome }\end{array}$ & 1 & 99 & DEMIDVIAVTKGHGFQGVTSR \\
\hline \multirow{8}{*}{131} & \multirow{8}{*}{25.66} & \multirow{8}{*}{ Q4WT69 } & \multirow{8}{*}{2.7 .2 .3} & \multirow{8}{*}{$44.76 / 6.31$} & \multirow{8}{*}{ AFUA_1G10350 } & \multirow{8}{*}{ A. fumigatus } & \multirow{8}{*}{ Phosphoglycerate kinase } & \multirow{8}{*}{$\begin{array}{l}\text { Phosphoglycerate } \\
\text { kinase activity }\end{array}$} & \multirow{8}{*}{8} & 99 & ALESPSRPFLAILGGSK \\
\hline & & & & & & & & & & 99 & ASGGQVILLENLR \\
\hline & & & & & & & & & & 99 & FHPEEEGSYKDEEGK \\
\hline & & & & & & & & & & 99 & FHPEEEGSYKDEEGKK \\
\hline & & & & & & & & & & 99 & GLTALGDIYINDAFGTAHR \\
\hline & & & & & & & & & & 99 & IGNSLFDEAGSK \\
\hline & & & & & & & & & & 99 & IVLPVDYITADKFSADAK \\
\hline & & & & & & & & & & 99 & YSLKPVVPELEK \\
\hline \multirow{7}{*}{132} & \multirow{7}{*}{14.52} & \multirow{7}{*}{ Q4WDF5 } & \multirow{7}{*}{-} & \multirow{7}{*}{$54.18 / 7.18$} & \multirow{7}{*}{ AFUA_6G04570 } & \multirow{7}{*}{ A. fumigatus } & \multirow{7}{*}{$\begin{array}{l}\text { Translation elongation } \\
\text { factor eEF-1 subunit } \\
\text { gamma, putative }\end{array}$} & \multirow{7}{*}{$\begin{array}{l}\text { Translation elongation } \\
\text { factor activity }\end{array}$} & \multirow{7}{*}{7} & 99 & AVVPSPVFAEEAIK \\
\hline & & & & & & & & & & 99 & EYPHVDGHVFK \\
\hline & & & & & & & & & & 99 & HLTANTYLVGER \\
\hline & & & & & & & & & & 99 & ITLADYFGASLLTR \\
\hline & & & & & & & & & & 99 & TKQDYAAILR \\
\hline & & & & & & & & & & 98 & QDYAAILR \\
\hline & & & & & & & & & & 97 & LYGLPENGR \\
\hline 141 & 5.65 & Q4WEU3 & 1.10 .2 .2 & $48.09 / 8.89$ & AFUA_5G04210 & A. fumigatus & $\begin{array}{c}\text { Ubiquinol-cytochrome } \mathrm{C} \\
\text { reductase complex core } \\
\text { protein 2, putative }\end{array}$ & $\begin{array}{l}\text { Ubiquinolcytochrome-C } \\
\text { reductase activity }\end{array}$ & 2 & 99 & $\begin{array}{c}\text { ATQGFSQVR } \\
\text { SNIAIVGSGSSTAEVSR }\end{array}$ \\
\hline
\end{tabular}


Table 1. Cont.

\begin{tabular}{|c|c|c|c|c|c|c|c|c|c|c|c|}
\hline Spot & $\begin{array}{l}\text { Cov. } \\
(95 \%) \\
\end{array}$ & $\begin{array}{l}\text { Accession } \\
\text { Number }\end{array}$ & $\begin{array}{c}\text { EC } \\
\text { Number }\end{array}$ & $\begin{array}{c}\text { Theorical } \\
M_{\mathrm{r}} / \mathrm{PI}\end{array}$ & Orf & Organism & Name & Function & Peptides & Conf. & Sequence \\
\hline 178 & 2.99 & В0XM32 & - & $56.40 / 6.84$ & AFUB_000800 & A. fumigatus & Cytochrome P450 & $\begin{array}{l}\text { Oxidoreductase activity, } \\
\text { acting on paired donors, } \\
\text { with incorporation or } \\
\text { reduction of } \\
\text { molecular oxygen } \\
\end{array}$ & 1 & 99 & LLSDQFAGFPSVNSR \\
\hline $\begin{array}{l}176, \\
180\end{array}$ & 22.15 & Q4WQK8 & - & $34.99 / 6.06$ & AFUA_4G13170 & A. fumigatus & $\begin{array}{l}\text { G-protein comlpex beta } \\
\text { subunit } \mathrm{CpcB}\end{array}$ & Cell signaling & 5 & $\begin{array}{l}99 \\
99 \\
99 \\
99 \\
99\end{array}$ & $\begin{array}{c}\text { VDELKPEFIEK } \\
\text { HLYSLHAGDEIHALVFSPNR } \\
\text { LWELATGETTR } \\
\text { TFVGHTSDVLSVSFSADNR } \\
\text { TLIIWNLTR } \\
\end{array}$ \\
\hline A & 3.29 & Q4WGN6 & 3.6.3.- & $117.77 / 5.84$ & AFUA_7G05660 & A. fumigatus & $\begin{array}{l}\text { Translation elongation } \\
\text { factor eEF-3 }\end{array}$ & $\begin{array}{c}\text { Translation elongation } \\
\text { factor activity }\end{array}$ & 3 & $\begin{array}{l}99 \\
99 \\
97\end{array}$ & $\begin{array}{c}\text { FLDNVIQHVVHYER } \\
\text { TFEGGVVIITHSR } \\
\text { LEEFGFLR }\end{array}$ \\
\hline B & 5.87 & Q4WX09 & - & $71.15 / 6.50$ & AFUA_3G07810 & A. fumigatus & $\begin{array}{l}\text { Succinate dehydrogenase } \\
\text { subunit Sdh1, putative }\end{array}$ & $\begin{array}{l}\text { Eectron transport chain; } \\
\text { tricarboxylic acid cycle }\end{array}$ & 3 & $\begin{array}{l}99 \\
99 \\
99 \\
\end{array}$ & $\begin{array}{l}\text { AHHTVLATGGYGR } \\
\text { KPHGEINLGYR } \\
\text { GIIAYNQEDGTLHR }\end{array}$ \\
\hline $\mathrm{C}$ & 5.45 & Q4X1P0 & - & $61.95 / 5.53$ & AFUA_2G09290 & A. fumigatus & $\begin{array}{l}\text { Antigenic mitochondrial } \\
\text { protein HSP60, putative }\end{array}$ & $\begin{array}{c}\text { Cellular response to } \\
\text { temperature stimulus; } \\
\text { protein refolding }\end{array}$ & 2 & $\begin{array}{l}99 \\
99\end{array}$ & $\begin{array}{l}\text { AITLQDKFENLGAR } \\
\text { ISAVQDIIPALEASTTLR }\end{array}$ \\
\hline
\end{tabular}


Table 1. Cont.

\begin{tabular}{|c|c|c|c|c|c|c|c|c|c|c|c|}
\hline Spot & $\begin{array}{c}\text { Cov. } \\
(95 \%) \\
\end{array}$ & $\begin{array}{c}\text { Accession } \\
\text { Number }\end{array}$ & $\begin{array}{c}\text { EC } \\
\text { Number }\end{array}$ & $\begin{array}{c}\text { Theorical } \\
M_{\mathrm{r}} / \mathrm{PI}\end{array}$ & Orf & Organism & Name & Function & Peptides & Conf. & Sequence \\
\hline \multirow{9}{*}{$\mathrm{D}$} & \multirow{9}{*}{20.81} & \multirow{9}{*}{ Q4WV25 } & \multirow{9}{*}{ 3.6.3.14 } & \multirow{9}{*}{$55.62 / 5.30$} & \multirow{9}{*}{ AFUA_5G10550 } & \multirow{9}{*}{ A. fumigatus } & \multirow{9}{*}{ ATP synthase subunit beta } & \multirow{9}{*}{ ATP catabolic process } & \multirow{9}{*}{9} & 99 & DTGAPIKIPVGPGTLGR \\
\hline & & & & & & & & & & 99 & FTQAGSEVSALLGR \\
\hline & & & & & & & & & & 99 & IPVGPGTLGR \\
\hline & & & & & & & & & & 99 & IVGEEHYAVATR \\
\hline & & & & & & & & & & 99 & IVNVTGDPIDER \\
\hline & & & & & & & & & & 99 & LVLEVSQHLGENVVR \\
\hline & & & & & & & & & & 99 & VALTGLTIAEYFR \\
\hline & & & & & & & & & & 99 & VALVFGQMNEPPGAR \\
\hline & & & & & & & & & & 99 & VVDLLAPYAR \\
\hline \multirow{7}{*}{$\mathrm{E}$} & \multirow{7}{*}{23.15} & \multirow{7}{*}{ Q4WX43 } & \multirow{7}{*}{ 3.6.4.13 } & \multirow{7}{*}{$45.78 / 5.05$} & \multirow{7}{*}{ AFUA_3G08160 } & \multirow{7}{*}{ A. fumigatus } & \multirow{7}{*}{$\begin{array}{l}\text { ATP-dependent RNA } \\
\text { helicase eIF4A }\end{array}$} & & \multirow{7}{*}{7} & 99 & ALQEGPQVVVGTPGR \\
\hline & & & & & & & & Complex eIF4F & & 99 & DFTVSAMHGDMEQAQR \\
\hline & & & & & & & & subunit-involved in the & & 99 & GCQALILAPTR \\
\hline & & & & & & & & "cap" recognition; & & 99 & GVAINFVTADDVR \\
\hline & & & & & & & & necessary to mRNA & & 99 & GVYAYGFERPSAIQQR \\
\hline & & & & & & & & binding to ribossome & & 99 & MFILDEADEMLSR \\
\hline & & & & & & & & & & 99 & VLIATDLLAR \\
\hline \multirow{5}{*}{$\mathrm{F}$} & \multirow{5}{*}{14.41} & \multirow{5}{*}{ Q4WNQ8 } & \multirow{5}{*}{-} & \multirow{5}{*}{$49.37 / 5.79$} & \multirow{5}{*}{ AFUA_4G06620 } & \multirow{5}{*}{ A. fumigatus } & & \multirow{5}{*}{ Oxidoreductase activity } & \multirow{5}{*}{5} & 99 & AANAGGVAVSGLEMAQNSAR \\
\hline & & & & & & & Glutamate dehydrogenase & & & 99 & FLGFEQIFK \\
\hline & & & & & & & -Glu/Leu/Phe/Val & & & 99 & VVWEDDNHQVQINR \\
\hline & & & & & & & dehydrogenase & & & 99 & YIEGARPWVHVGK \\
\hline & & & & & & & & & & 99 & EIGFLFGQYR \\
\hline
\end{tabular}


Table 1. Cont.

\begin{tabular}{|c|c|c|c|c|c|c|c|c|c|c|c|}
\hline Spot & $\begin{array}{l}\text { Cov. } \\
(95 \%) \\
\end{array}$ & $\begin{array}{c}\text { Accession } \\
\text { Number }\end{array}$ & $\begin{array}{c}\text { EC } \\
\text { Number }\end{array}$ & $\begin{array}{c}\text { Theorical } \\
M_{\mathrm{r}} / \mathbf{P I}\end{array}$ & Orf & Organism & Name & Function & Peptides & Conf. & Sequence \\
\hline \multirow{6}{*}{ G } & \multirow{6}{*}{18.06} & \multirow{6}{*}{ Q4WY39 } & \multirow{6}{*}{4.1 .2 .13} & \multirow{6}{*}{$39.79 / 5.55$} & \multirow{6}{*}{ AFUA_3G11690 } & \multirow{6}{*}{ A. fumigatus } & \multirow{6}{*}{$\begin{array}{c}\text { Fructose-bisphosphate } \\
\text { aldolase, class II }\end{array}$} & \multirow{6}{*}{$\begin{array}{l}\text { Fructose-bisphosphate } \\
\text { aldolase activity; } \\
\text { zinc ion binding }\end{array}$} & \multirow{6}{*}{6} & 99 & ASIAGSIAAAHYIR \\
\hline & & & & & & & & & & 99 & KSGVIVGDDVLR \\
\hline & & & & & & & & & & 99 & LFEYAQEK \\
\hline & & & & & & & & & & 99 & RVQVALEDFNTAGQL \\
\hline & & & & & & & & & & 99 & SGVIVGDDVLR \\
\hline & & & & & & & & & & 99 & VNLDTDMQYAYMSGVR \\
\hline \multirow{8}{*}{ I } & \multirow{3}{*}{6.37} & \multirow{3}{*}{ Q4WQ26 } & \multirow{3}{*}{-} & \multirow{3}{*}{$42.35 / 5.68$} & \multirow{3}{*}{ AFUA_4G11330 } & \multirow{3}{*}{ A. fumigatus } & \multirow{3}{*}{ Aha1 domain family } & ATPase activator & \multirow{3}{*}{3} & \multirow{3}{*}{99} & QNWDVYYVR \\
\hline & & & & & & & & activity-Response & & & VAVNTTTVTASDEFR \\
\hline & & & & & & & & to stress & & & QNWDVYYVR \\
\hline & \multirow{5}{*}{18.71} & \multirow{5}{*}{ Q4WQK3 } & \multirow{5}{*}{ 6.3.1.2 } & \multirow{5}{*}{$39.90 / 5.48$} & \multirow{5}{*}{ AFUA_4G13120 } & \multirow{5}{*}{ A. fumigatus } & \multirow{5}{*}{ Glutamine synthetase } & \multirow{5}{*}{$\begin{array}{l}\text { Glutamate-ammonia } \\
\text { ligase activity-Glutamine } \\
\text { biosynthetic process }\end{array}$} & \multirow{5}{*}{5} & \multirow{5}{*}{99} & DIVEAHYR \\
\hline & & & & & & & & & & & FSYGVADR \\
\hline & & & & & & & & & & & GDWNGAGLHTNVSTAATR \\
\hline & & & & & & & & & & & GGFPGAQGPYYCGVGTGK \\
\hline & & & & & & & & & & & HNEHIAVYGEGNEER \\
\hline \multirow{7}{*}{ K } & & & & & & & & & & & AGDNSGLLLR \\
\hline & & & & & & & & & & & GITISTAHIEFSTDSR \\
\hline & & & & & & & & Translation elongation & & & GLANFLEYGAIDKAPEER \\
\hline & 23.86 & Q8TGG6 & - & $48.29 / 6.69$ & AfA14E5.05 & A. fumigatus & Elongation factor $\mathrm{Tu}$ & factor activity-Protein & 7 & 99 & HYAHVDCPGHADYIK \\
\hline & & & & & & & & biosynthesis & & & TADEAADLSFPDGDQSR \\
\hline & & & & & & & & & & & THHPVAAEAGQR \\
\hline & & & & & & & & & & & TKPHVNIGTIGHVDHGK \\
\hline & & & & & & & Pyruvate dehydrogenase & Pyruvate dehydrogenase & & & ILFEDIYVR \\
\hline $\mathrm{L}$ & 4.44 & Q4WJ75 & 1.2.4.1 & $41.48 / 6.36$ & AFUA_1G06960 & A. fumigatus & $\begin{array}{l}\text { E1 component } \\
\text { subunit alpha }\end{array}$ & $\begin{array}{l}\text { (acetyl-transferring) } \\
\text { activity-Glycolytic process }\end{array}$ & 2 & 99 & SIIGELLGR \\
\hline
\end{tabular}


Table 1. Cont.

\begin{tabular}{|c|c|c|c|c|c|c|c|c|c|c|c|}
\hline Spot & $\begin{array}{c}\text { Cov. } \\
(95 \%) \\
\end{array}$ & $\begin{array}{c}\text { Accession } \\
\text { Number }\end{array}$ & $\begin{array}{c}\text { EC } \\
\text { Number }\end{array}$ & $\begin{array}{c}\text { Theorical } \\
M_{\mathrm{r}} / \mathbf{P I}\end{array}$ & Orf & Organism & Name & Function & Peptides & Conf. & Sequence \\
\hline \multirow{7}{*}{$\mathrm{N}$} & \multirow{7}{*}{14.76} & \multirow{7}{*}{ Q4WEU5 } & \multirow{7}{*}{-} & \multirow{7}{*}{$52.11 / 8.69$} & \multirow{7}{*}{ AFUA_5G04230 } & \multirow{7}{*}{ A. fumigatus } & \multirow{7}{*}{ Citrate synthase } & & \multirow{7}{*}{4} & \multirow{7}{*}{99} & CLVWEGSVLDSEEGIR \\
\hline & & & & & & & & Citrate $(\mathrm{Si})$-synthase & & & FIEELIDR \\
\hline & & & & & & & & activity - Tricarboxylic & & & ALGAPIERPK \\
\hline & & & & & & & & acid cycle/Cellular & & & ALGVLPQLIIDR \\
\hline & & & & & & & & carbohydrate & & & DLSAEWAAR \\
\hline & & & & & & & & metabolic process & & & FIEELIDR \\
\hline & & & & & & & & & & & VIGEVTLDQAYGGAR \\
\hline 0 & 502 & O4WWD5 & $3-2$ & $5302 / 547$ & АFUA $3 G 05450$ & 4 fumigatus & Glutamate & Carboxypeptidase, & 2 & 00 & EHLDLPPVVIAR \\
\hline 0 & 5.02 & Q4WWDS & 3....-- & $53.02 / 5.4 /$ & AFUA_3U05450 & A. Jumtgatus & carboxypeptidase, putative & Hydrolase Protease & 2 & 99 & QVDELSNSFIDR \\
\hline \multirow{5}{*}{$\mathrm{P}$} & \multirow{5}{*}{8.85} & \multirow{5}{*}{ Q4WYW4 } & \multirow{5}{*}{1.1 .1 .86} & \multirow{5}{*}{$56.35 / 9.32$} & \multirow{5}{*}{ AFUA_3G14490 } & \multirow{5}{*}{ A. fumigatus } & \multirow{5}{*}{$\begin{array}{l}\text { Ketol-acid } \\
\text { reductoisomerase }\end{array}$} & Ketol-acid & \multirow{5}{*}{3} & \multirow{5}{*}{99} & DQGLNVIVGVR \\
\hline & & & & & & & & reductoisomerase & & & EVYSDLYGER \\
\hline & & & & & & & & activity-branched-chain & & & \\
\hline & & & & & & & & amino acid & & & TLYFSHGFSPVFK \\
\hline & & & & & & & & biosynthetic process & & & \\
\hline
\end{tabular}

Cov. $=$ Coverage EC number $=$ Enzyme Commission number; $M_{\mathrm{r}}=$ Molecular weight range in $\mathrm{kDa} ; \mathrm{PI}=$ Isoelectric point; Conf. $=$ Confidence. 
Figure 2. 2-D Western immunoblot of proteins extracts of $A$. fumigatus germlings from the pool of patient's sera classified as (A) proven/hospital 1; (B) probable; (C) proven/hospital 2; or (D) other-mycosis. The identified antigenic proteins are indicated with the accession number (UniProtKB).

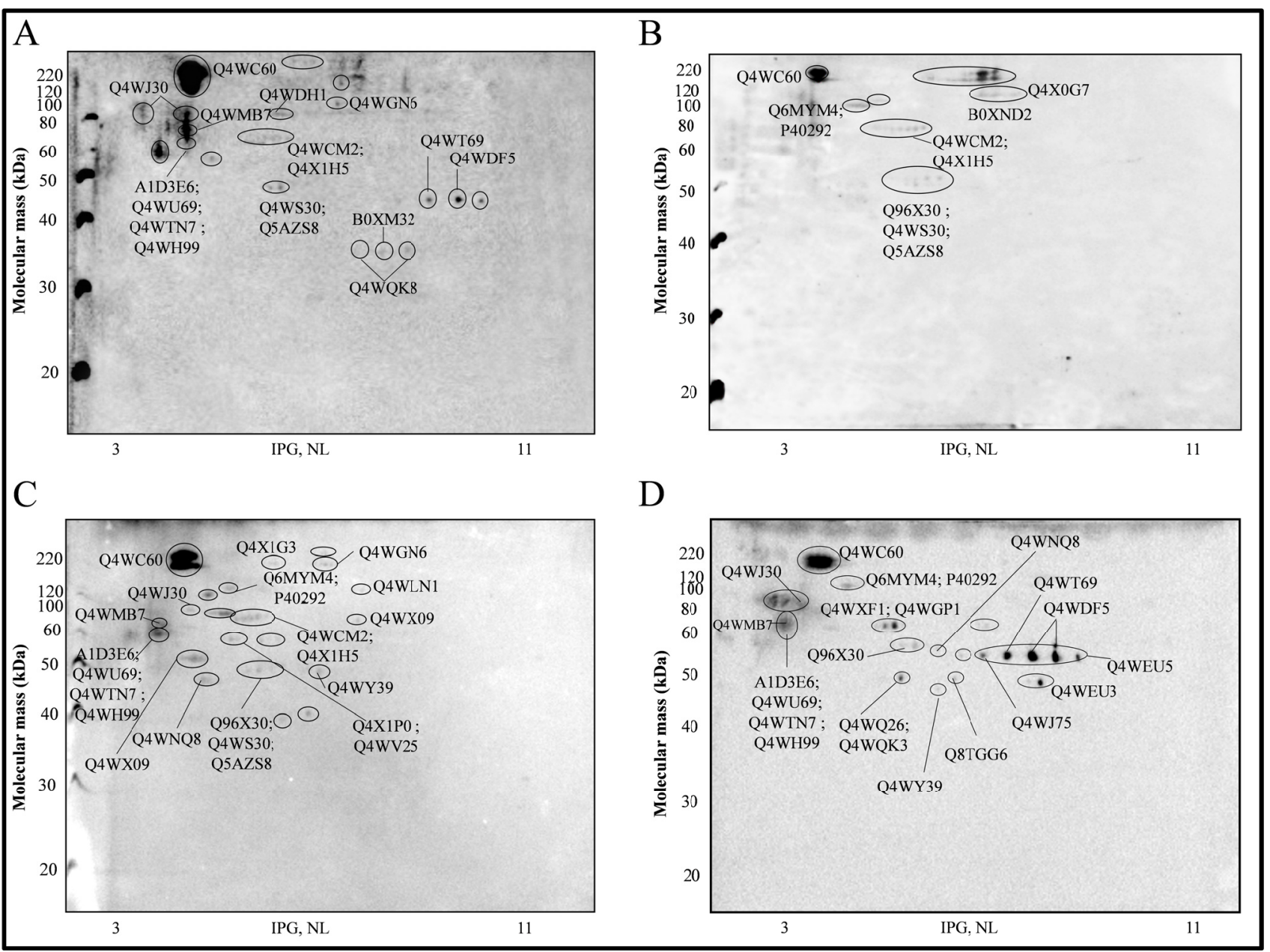

Table 2. Antigenic proteins revealed using the different pools of sera.

\begin{tabular}{|c|c|c|c|c|c|c|}
\hline Spot & Name & $\begin{array}{c}\text { Proven- } \\
\text { Hospital } 1\end{array}$ & $\begin{array}{c}\text { Proven- } \\
\text { Hospital } 2\end{array}$ & Probable & $\begin{array}{c}\text { Other } \\
\text { Mycoses }\end{array}$ & Control \\
\hline 1 & Probable $\beta$-glucosidase btgE & $\mathrm{X}$ & $\mathrm{X}$ & $\mathrm{X}$ & $\mathrm{X}$ & $\mathrm{X}$ \\
\hline 8 & Carbamoyl-phosphate synthase, large subunit & & $\mathrm{X}$ & & & \\
\hline $\begin{array}{l}11,12,13 \\
14,15\end{array}$ & Translation elongation factor EF-2 subunit, putative & & & $\mathrm{X}$ & & \\
\hline 13,15 & Polyadenylate-binding protein & & & $\mathrm{X}$ & & \\
\hline \multirow[b]{2}{*}{24} & Heat shock protein Hsp88, putative & $\mathrm{X}$ & $\mathrm{X}$ & $\mathrm{X}$ & $\mathrm{X}$ & \\
\hline & $\begin{array}{l}\text { Heat shock protein } 90-\text { Heat shock protein hsp1 } \\
\qquad(\text { Asp f 12) }\end{array}$ & & $\mathrm{X}$ & $\mathrm{X}$ & $\mathrm{X}$ & \\
\hline 26 & $\begin{array}{l}\text { Bifunctional purine biosynthetic protein Ade1, } \\
\text { putative }\end{array}$ & $\mathrm{X}$ & & & & \\
\hline $35,36,37$ & Mitochondrial aconitate hydratase, putative & & $\mathrm{X}$ & & & \\
\hline 46,47 & Molecular chaperone Hsp70 & $\mathrm{X}$ & $\mathrm{X}$ & & $\mathrm{X}$ & $\mathrm{X}$ \\
\hline
\end{tabular}


Table 2. Cont.

\begin{tabular}{|c|c|c|c|c|c|c|}
\hline Spot & Name & $\begin{array}{c}\text { Proven- } \\
\text { Hospital } 1 \\
\end{array}$ & $\begin{array}{c}\text { Proven- } \\
\text { Hospital } 2 \\
\end{array}$ & Probable & $\begin{array}{c}\text { Other } \\
\text { Mycoses }\end{array}$ & Control \\
\hline 59 & Zinc finger protein ZPR1 & $\mathrm{X}$ & $\mathrm{X}$ & & $\mathrm{X}$ & \\
\hline 61,62 & Hsp70 chaperone (HscA), putative & $\mathrm{X}$ & $\mathrm{x}$ & $\mathrm{X}$ & & \\
\hline $\begin{array}{c}63,64 \\
65\end{array}$ & Mitochondrial Hsp70 chaperone (Ssc70), putative & $\mathrm{X}$ & $\mathrm{x}$ & $\mathrm{X}$ & & \\
\hline \multirow{4}{*}{71} & Protein phosphatase $2 \mathrm{C}$, putative & $\mathrm{X}$ & $\mathrm{x}$ & & $\mathrm{x}$ & $\mathrm{x}$ \\
\hline & $60 \mathrm{~S}$ ribosome biogenesis protein Sqt1, putative & $\mathrm{X}$ & $\mathrm{X}$ & & $\mathrm{X}$ & $\mathrm{X}$ \\
\hline & Nucleosome assembly protein Nap1, putative & $X$ & $X$ & & $X$ & $X$ \\
\hline & Protein disulfide isomerase Pdi1, putative & $\mathrm{X}$ & $\mathrm{X}$ & & $\mathrm{X}$ & $\mathrm{X}$ \\
\hline \multirow{2}{*}{73,74} & $\begin{array}{c}\text { Phosphoglycerate mutase, } \\
\text { 2,3-bisphosphoglycerate-independent }\end{array}$ & & & & $\mathrm{X}$ & \\
\hline & $\begin{array}{l}\text { Pyruvate dehydrogenase complex, dihydrolipoamide } \\
\text { acetyltransferase component, putative }\end{array}$ & & & & $\mathrm{X}$ & \\
\hline 114,117 & Enolase (Asp f 22) & & $\mathrm{x}$ & $\mathrm{X}$ & $\mathrm{X}$ & \\
\hline 119,120 & $\begin{array}{l}\text { Mitochondrial processing peptidase } \beta \\
\text { subunit, putative }\end{array}$ & $\mathrm{X}$ & $\mathrm{x}$ & $\mathrm{X}$ & & \\
\hline 120 & RL3_NEUCR 60S ribosomal protein L3 & $\mathrm{X}$ & $\mathrm{x}$ & $\mathrm{X}$ & & \\
\hline 131 & Phosphoglycerate kinase & $\mathrm{X}$ & & & $\mathrm{X}$ & $\mathrm{X}$ \\
\hline 132,133 & $\begin{array}{c}\text { Translation elongation factor eEF-1 subunit } \gamma, \\
\text { putative }\end{array}$ & $\mathrm{X}$ & & & $\mathrm{X}$ & $\mathrm{X}$ \\
\hline 140,141 & $\begin{array}{l}\text { Ubiquinol-cytochrome } \mathrm{C} \text { reductase complex core } \\
\text { protein 2, putative }\end{array}$ & & & & $\mathrm{X}$ & \\
\hline 178 & Cytochrome P450 & $\mathrm{X}$ & & & & \\
\hline 176,180 & G-protein comlpex beta subunit $\mathrm{CpcB}$ & $\mathrm{x}$ & & & & \\
\hline A & Translation elongation factor eEF-3 & $\mathrm{X}$ & $\mathrm{x}$ & & & \\
\hline $\mathrm{B}$ & Succinate dehydrogenase subunit Sdh1, putative & & $\mathrm{x}$ & & & \\
\hline $\mathrm{C}$ & Antigenic mitochondrial protein $\mathrm{HSP} 60$, putative & & $\mathrm{X}$ & & & \\
\hline $\mathrm{D}$ & ATP synthase subunit $\beta$ & & $\mathrm{X}$ & & & \\
\hline $\mathrm{E}$ & ATP-dependent RNA helicase eIF4A & & $\mathrm{x}$ & & & \\
\hline $\mathrm{F}$ & $\begin{array}{c}\text { Glutamate dehydrogenase—Glu/Leu/Phe/Val } \\
\text { dehydrogenase }\end{array}$ & & $\mathrm{x}$ & & $\mathrm{X}$ & \\
\hline G & Fructose-bisphosphate aldolase, class II & & $\mathrm{X}$ & & $\mathrm{X}$ & \\
\hline I & Aha1 domain family & & & & $\mathrm{X}$ & \\
\hline I & Glutamine synthetase & & & & $\mathrm{X}$ & \\
\hline $\mathrm{K}$ & Elongation factor $\mathrm{Tu}$ & & & & $\mathrm{x}$ & \\
\hline $\mathrm{L}$ & $\begin{array}{l}\text { Pyruvate dehydrogenase E1 component } \\
\text { subunit } \alpha\end{array}$ & & & & $\mathrm{X}$ & \\
\hline $\mathrm{N}$ & Citrate synthase & & & & $\mathrm{X}$ & \\
\hline $\mathrm{O}$ & Glutamate carboxypeptidase, putative & & & & & $\mathrm{x}$ \\
\hline $\mathrm{P}$ & Ketol-acid reductoisomerase & & & & & $\mathrm{x}$ \\
\hline
\end{tabular}

A total of fourteen antigenic proteins were exclusively revealed by sera of patients with proven aspergillosis, as shown in Table 2 (grey lines). Among these, four proteins were also recognized by 
pool of sera classified as probable by the EORTC/MSG criteria. Five out of fourteen proteins were positively recognized by the pool of patients with proven aspergillosis from both Hospital 1 and 2 . Some of these identified antigens had also been described in other reports based on assays with the sera of immunized rabbits, mice and of patients with the clinical suspicion of allergic bronchopulmonary aspergillosis [20,21,23,28,32]. To our knowledge, this work is the first to describe four antigens: eEF-3, eIF4A, cytochrome P450 and Ade1, which are putative candidates for diagnostic utility.

\subsection{BLAST Analysis}

The fourteen antigens revealed from the immunoproteome of the sera from patients with proven invasive aspergillosis $(n=12)$ were selected as putative candidates for the diagnosis of invasive aspergillosis. Their protein sequences were compared with human proteins via BLAST analyses to ensure their potential specificity for $A$. fumigatus and cross-reactivity with human proteins. Our results showed that only two antigenic proteins, cytochrome P450 and eEF-3, had no homology with human proteins.

As mentioned previously, the diagnosis of invasive aspergillosis can be confused with a range of other invasive fungal infections [33-37]. In this context, we also compared (via BLAST analysis) the sequences of the two above-described proteins with proteins of Rizophus spp. and other fungi from the Mucorales order; Penicillium spp., Paracoccidioides brasiliensis, Fusarium spp., and Paecilomyces spp., as described in the methodology section. The results shown in Table 3 indicate that both cytochrome P450 and eEF-3 can be putative markers for the selective diagnosis of $A$. fumigatus infections.

Table 3. BLAST analysis of the two main antigens identified against the protein sequences of etiological agents of other invasive fungal infections.

\begin{tabular}{|c|c|c|c|}
\hline Microorganisms & Parameters & Cytochrome P450 & eEF-3 \\
\hline \multirow{4}{*}{ Mucorales } & Score & 56.2 & 23.9 \\
\hline & E-value & $8 \times 10^{-11}$ & 2.2 \\
\hline & Identity & $25 \%$ & $26 \%$ \\
\hline & $\begin{array}{c}\text { Protein homology } \\
\text { (organism) }\end{array}$ & $\begin{array}{c}\text { Cytochrome P450 } 51 \\
\text { (Cunninghamella elegans) }\end{array}$ & $\begin{array}{c}\text { Glyceraldehyde-3-phosphate } \\
\text { dehydrogenase (Rhizomucor miehei) }\end{array}$ \\
\hline \multirow{4}{*}{ Penicillium } & Score & 45.1 & 27.3 \\
\hline & E-value & $6 \times 10^{-7}$ & 0.57 \\
\hline & Identity & $23 \%$ & $56 \%$ \\
\hline & $\begin{array}{c}\text { Protein homology } \\
\text { (organism) }\end{array}$ & $\begin{array}{l}\text { Eburicol 14- } \alpha \text {-demethylase } \\
\text { (Penicillium chrysogenum) }\end{array}$ & $\begin{array}{c}\text { Peroxisomal biogenesis factor } 6 \\
(\text { Penicillium chrysogenum })\end{array}$ \\
\hline \multirow{4}{*}{ P. brasiliensis } & Score & 48.1 & 26.6 \\
\hline & E-value & 0.64 & 0.51 \\
\hline & Identity & $56 \%$ & $33 \%$ \\
\hline & $\begin{array}{c}\text { Protein homology } \\
\text { (organism) }\end{array}$ & Translation factor GUF1 & $\begin{array}{c}\text { Probable Xaa-Pro } \\
\text { aminopeptidase PADG }\end{array}$ \\
\hline
\end{tabular}


Table 3. Cont.

\begin{tabular}{|c|c|c|c|}
\hline Microorganisms & Parameters & Cytochrome P450 & eEF-3 \\
\hline \multirow{4}{*}{ Rhizopus } & Score & 19.6 & 43.5 \\
\hline & E-value & 8.8 & 2.7 \\
\hline & Identity & $67 \%$ & $26 \%$ \\
\hline & $\begin{array}{l}\text { Protein homology } \\
\text { (organism) }\end{array}$ & $\begin{array}{l}\text { Rhizopuspepsin-2 } \\
\text { (Rhizopus niveus) }\end{array}$ & $\begin{array}{c}\text { Peptidyl-prolyl cis-trans isomerase } \\
\text { cyp11 (Rhizopus delemar) }\end{array}$ \\
\hline \multirow{4}{*}{ Fusarium } & Score & 211 & 112 \\
\hline & E-value & $5 \times 10^{-63}$ & $6 \times 10^{-6}$ \\
\hline & Identity & $32 \%$ & $35 \%$ \\
\hline & $\begin{array}{c}\text { Protein homology } \\
\text { (organism) }\end{array}$ & $\begin{array}{l}\text { Cytochrome } \mathrm{P} 450503 \mathrm{~A} 1 \\
\text { (Fusarium proliferatum) }\end{array}$ & $\begin{array}{l}\text { Iron-sulfur clusters transporter } \\
\text { ATM1 (Fusarium graminearum) }\end{array}$ \\
\hline \multirow{4}{*}{ Paecilomyces } & Score & - & - \\
\hline & E-value & - & - \\
\hline & Identity & - & - \\
\hline & $\begin{array}{c}\text { Protein homology } \\
\text { (organism) } \\
\end{array}$ & No match & No match \\
\hline
\end{tabular}

The cytochrome P450 superfamily is made up of monooxygenases that play key roles in a range of biochemical processes from catalysis to xenobiotic detox and degradation; cytochrome P450 is found in every living form [38]. In general, cytochrome P450 isoforms have being described as essential for the membrane ergosterol biosynthesis, and some isoforms are involved in the production of aflatoxin in A. parasiticus [39-41]. In A. fumigatus, triazole resistance is often related to mutations in a gene that encodes a cytochrome P450 isoform, the cyp51 gene [42-44]. Although the secondary structures of the proteins of the cytochrome P450 superfamily are well conserved, there is a low homology among the primary amino acid sequences of different species [45-48]. These data are consistent with the result of our BLAST analysis that shows the low homology of the identified A. fumigatus cytochrome $\mathrm{P} 450$ found in this study with proteins of other fungi (Table 3). The cytochrome P450 identified in this study is predicted in the A. fumigatus genome but has no characterized function. To our knowledge, this is the first report showing the antigenic diagnostic potential of an $A$. fumigatus cytochrome P450.

The most promising antigen was the translation elongation factor eEF-3. This protein showed the lowest sequence homology in the BLAST analysis (Table 3). The translation process functions in a series highly regulated steps that are catalyzed by the eukaryotic initiation factors [49]. In general, the process is highly conserved from bacteria to mammals: the eEF-1 is incumbent on delivering the aminoacyl-tRNA to the ribosomal A-site [50], and the eEF-2 has a translocase activity [51]. However, another factor is required in fungi (an ATPase factor, namely eEF3). This requirement is unique in fungi ribosomes. This fungal-specific protein is absent in mammalian cells and has already being described by our group as a putative drug target in A. fumigatus [27]. The eEF-3 is an ATPase of the ATP binding cassette (ABC) family member [52]. The majority of this superfamily's members are integral membrane transporters that are involved in the import or export of diverse substrates across lipid bilayers [53]. However, eEF-3 lacks the transmembrane domain because it is a soluble factor with two $\mathrm{ABC}$ domains arranged in tandem. One of these domains carries a unique chromodomain-like 
insertion that is hypothesized to play a significant role in its binding to the ribosome [54]. A recent study showed that mutations in the chromodomain-like insertion of eEF-3 resulted in reduced growth rate and slower translation elongation. These mutations also compromised the ribosome-stimulated ATPase activity of eEF3, strongly suggesting that it exerts an allosteric effect on the hydrolytic activity of eEF3 [55]. These features contributed to the overexpression of eEF-3 in the first steps of A. fumigatus filamentation (germlings), strengthening the hypothesis that this protein may be a good drug target [31].

Our previous studies showed that this protein was found to be overexpressed up to eight-fold on the surface of the germlings compared with mature A. fumigatus hyphae [31]. In this study, the eEF-3 factor was identified as an antigenic protein of $A$. fumigatus recognized by the sera of patients with proven invasive aspergillosis. Taken together, these observations strongly suggest that in addition to being a putative drug target, the identified A. fumigatus eEF-3 factor can also be a promising candidate for the diagnosis of invasive aspergillosis.

\section{Experimental Section}

\subsection{Fungal Strain and Culture Conditions}

The A. fumigatus strain used in this study was AF293, which was originally isolated at autopsy from a patient with IPA and kindly provided by Dr. Scott Filler of Harbor-UCLA Medical Center, University of California, CA, USA.

A. fumigatus was first grown in Sabouraud Agar (Difco, Detroit, MI, USA) roux flask for 7 days at $37{ }^{\circ} \mathrm{C}$. The conidia were than harvested using a cell scraper in the presence of PBS-Tween 20 $(0.01 \%)$. This suspension was vacuum-filtered using a Büchner filler with a nylon membrane (Sefar Nitex 03-28/17, 7, Sefar Inc., Heiden, Switzerland) to remove hyphae fragments. A ratio of $10^{7}$ conidia/mL was then incubated in Sabouraud Broth (Difco, Maryland, MD, USA) in a 500-mL flask on a shaker at $37^{\circ} \mathrm{C}$ and $150 \mathrm{rpm}$ for $6 \mathrm{~h}$ to obtain the conidia germlings.

\subsection{Preparation of Germiling Conidia Protein Extract $\left(G T_{6 \mathrm{~h}}\right)$}

Conidia germling cells were submitted to chemical extraction [56] using protein extraction buffer containing Tris- $\mathrm{HCl} 25 \mathrm{mM}$, DTT $2 \mathrm{mM}$, PMSF $1 \mathrm{mM}$ and EDTA $5 \mathrm{mM}, \mathrm{pH}$ 8.5. The conidia germling cells were incubated with the protein extraction buffer in a ratio of $0.7 \mathrm{~g}$ of cells (wet weight) per $5 \mathrm{~mL}$ of buffer for $2 \mathrm{~h}$ at $4{ }^{\circ} \mathrm{C}$ under gentle agitation. The proteins extracted using this process were separated via centrifugation. The extract was precipitated with trichloroacetic acid/acetone [57] and re-suspended in rehydration buffer containing $7 \mathrm{M}$ urea, $2 \mathrm{M}$ thiourea and CHAPS 4\%. The protein concentration was determined using the Bradford method (Bio-Rad, Hercules, CA, USA) according to the manufacturer's recommendations. The absence of membrane leakage and consequently intracellular proteins or material derived from dead cells, in this type of extraction have been previously described [27].

\subsection{Patients and Control Subjects}

All of the serum samples of patients were obtained with informed patient consent and the permission of the local human ethics committee. All serum samples were classified according to the EORTC/MSG 
criteria [18]. Three serum samples of patients clinically diagnosed as proven and thirteen serum samples of patients clinically diagnosed as probable were obtained from the Bone Marrow Transplant Center of the National Institute of Cancer (INCA-Brazil), henceforth referred to as Hospital 1. More information about the characteristics of the patients from Hospital 1 is shown in Table 4. Nine serum samples of patients classified as "proven" for invasive aspergillosis were obtained from the Hospital das Clínicas of the Faculty of Medicine from the University of São Paulo (USP-Brazil), henceforth referred to as Hospital 2. Serum samples from patients with other fungal infections viz. histoplasmosis $(n=1)$, fusariosis $(n=3)$, cryptococcosis $(n=1)$ and paracoccidioidomycosis $(n=1)$ were also provided by Hospital 2. These patients had also underlying diseases similar to those found in the aspergillosis cases. As a negative control, sera from six patients with underlying diseases similar to the aspergillosis cases, such as acute myeloid leukemia $(n=2)$, non-Hodgkin lymphoma $(n=2)$, multiple myeloma $(n=1)$ and myelodysplastic syndrome $(n=1)$, were also provided by Hospital 2 . These patients did not receive antifungal treatment, presented no colonization by any fungal species and survived for at least 30 days. More information about the characteristics of the patients from Hospital 2 is shown in Table 5. The serum samples were pooled for the immunoproteome assays as follows: proven/hospital 1, proven/hospital 2, probable or other-mycosis.

Table 4. Additional information about patients from Hospital 1.

\begin{tabular}{|c|c|c|c|c|c|}
\hline Patient Hospital 1 & Gender & Age & $\begin{array}{c}\text { Underlying } \\
\text { Disease }\end{array}$ & Histopathology & $\begin{array}{c}\text { EORTC/MSG } \\
\text { Classification }\end{array}$ \\
\hline 1 & $\mathrm{M}$ & 10 & ALL/HSCT & - & Probable \\
\hline 2 & $\mathrm{~F}$ & 5 & MDS/HSCT & - & Probable \\
\hline 3 & $\mathrm{~F}$ & 39 & MDS/HSCT & - & Probable \\
\hline 4 & $\mathrm{~F}$ & 22 & $\mathrm{HL} / \mathrm{HSCT}$ & - & Probable \\
\hline 5 & M & 16 & ALL/HSCT & - & Probable \\
\hline 6 & M & 34 & $\mathrm{HL} / \mathrm{HSCT}$ & - & Probable \\
\hline 7 & M & 15 & ALL/HSCT & - & Probable \\
\hline 8 & $\mathrm{~F}$ & 53 & CML/HSCT & - & Probable \\
\hline 9 & M & 20 & ALL/HSCT & - & Probable \\
\hline 10 & M & 53 & $\mathrm{AA} / \mathrm{HSCT}$ & - & Probable \\
\hline 11 & M & 50 & AML/HSCT & - & Probable \\
\hline 12 & M & 9 & ALL/HSCT & - & Probable \\
\hline 13 & $\mathrm{~F}$ & 7 & ALL/HSCT & - & Probable \\
\hline 14 & $\mathrm{~F}$ & 29 & NHL/HSCT & A, fumigates (lung biopsy) & Proven \\
\hline 15 & $\mathrm{~F}$ & 11 & AML/HSCT & A, fumigates (lung biopsy) & Proven \\
\hline 16 & $\mathrm{~F}$ & 28 & AML/HSCT & A, flavus (lung biopsy) & Proven \\
\hline
\end{tabular}

EORTC/MSG = European Organization for Research and Treatment of Cancer (EORTC), Mycoses Study Group (MSG); ALL = Acute Lymphoblastic Leukemia; HSCT = Hematopoietic Stem Cell Transplantation; MDS = Myelodysplastic Syndrome; HL = Hodgkin Lymphoma; CML = Chronic Myeloid Leukemia; $\mathrm{AA}=$ Aplastic Anemia; AML = Acute Myeloid Leukemia; NHL = non-Hodgkin Lymphoma. 
Table 5. Additional information about patients from Hospital 2.

\begin{tabular}{|c|c|c|c|c|c|}
\hline $\begin{array}{c}\text { Patient } \\
\text { Hospital } 2\end{array}$ & Gender & Age & $\begin{array}{l}\text { Underlying } \\
\text { Disease }\end{array}$ & Histopathology & $\begin{array}{c}\text { EORTC/MSG } \\
\text { Classification }\end{array}$ \\
\hline 1 & $\mathrm{~F}$ & 19 & AML & Aspergillus sp. (necropsy) & Proven \\
\hline 2 & $\mathrm{~F}$ & 28 & AML/HSCT & Aspergillus sp. (necropsy) & Proven \\
\hline 3 & $\mathrm{~F}$ & 50 & $\mathrm{NHL} / \mathrm{HSCT}$ & Aspergillus sp. (lung biopsy) & Proven \\
\hline 4 & $\mathrm{~F}$ & 58 & ALL & Aspergillus sp. (necropsy) & Proven \\
\hline 5 & M & 26 & ALL & Aspergillus sp. (laryngeal biopsy) & Proven \\
\hline 6 & M & 58 & Lymphoma/HSCT & Aspergillus sp. (sinus biopsy) & Proven \\
\hline 7 & M & 39 & AML & Aspergillus sp. (sinus biopsy) & Proven \\
\hline 8 & M & 59 & NHL & Aspergillus sp. (lung biopsy) & Proven \\
\hline 9 & $\mathrm{~F}$ & 9 & $\begin{array}{c}\text { Fulminant } \\
\text { hepatitis/SOT }\end{array}$ & Aspergillus sp. (lung biopsy and necropsy) & Proven \\
\hline 10 & M & 35 & $\mathrm{AA} / \mathrm{HSCT}$ & Fusarium sp. (blood culture and skin biopsy) & Proven \\
\hline 11 & M & 17 & $\mathrm{AA} / \mathrm{HSCT}$ & Fusarium sp. (blood culture and skin biopsy) & Proven \\
\hline 12 & M & 51 & NHL/HSCT & Fusarium sp. (blood culture) & Proven \\
\hline 13 & $\mathrm{~F}$ & 49 & No & $\begin{array}{l}\text { Histoplasma sp. (lymph node biopsy and } \\
\text { immuno-histochemistry) }\end{array}$ & Proven \\
\hline 14 & M & 41 & No & $\begin{array}{l}\text { Paracoccidioides sp. (tracheal secretion } \\
\text { culture and direct mycroscopy of palatum) }\end{array}$ & Proven \\
\hline 15 & $\mathrm{~F}$ & 18 & SEL & $\begin{array}{l}\text { Cryptococcus neoformans var. gattii } \\
\text { (bronchoalveolar lavage culture) }\end{array}$ & Proven \\
\hline
\end{tabular}

\subsection{2-D SDS PAGE}

The focusing was performed using 75 or $400 \mu \mathrm{g}$ of GT6h protein and IPG strips (Immobiline DryStrip 3-11 NL, $18 \mathrm{~cm}$ ) with the addition of 1.2\% DeStreak and 1\% IPG buffer 3-11 (GE Healthcare, Piscataway, NJ, USA). Immobilized $\mathrm{pH}$-gradient strips were reduced $(1.5 \% \mathrm{w} / \mathrm{v}$ dithioerythritol) and alkylated (2.5\% $\mathrm{w} / \mathrm{v}$ iodocetamide) in equilibration buffer ( $6 \mathrm{M}$ urea, $50 \mathrm{mM}$ Tris- $\mathrm{HCl}$, $\mathrm{pH}$ 6.8, 30\% glycerol, 2\% SDS). Equilibrated strips were run on homogeneous 12\% polyacrylamide gels using a Protean II XL cell electrophoresis system (Bio-Rad, Hercules, CA, USA). The analytic gels were stained with silver [58], and preparative gels were stained using colloidal Coomassie [59] for protein identification.

\subsection{Western Immunoblot}

For the immunoblottings, the resolved proteins were transferred to nitrocellulose membranes using a Trans-Blot Cell system (Bio-Rad). The transblotted proteins on the membrane were checked with Ponceau, and each membrane was blocked with 5\% skim milk solution in $50 \mathrm{mM}$ Tris and $150 \mathrm{mM}$ $\mathrm{NaCl}$ containing $0.1 \%$ of Tween-20 (TBS-T). Then, the membranes were washed with $1 \%$ skim milk solution in TBS-T and incubated separately with each primary antibody (pools of sera: proven/hospital 1, proven/hospital 2, probable, other-mycosis, control) diluted in TBS-T at a 1:500 ratio for two hours at 
$4{ }^{\circ} \mathrm{C}$ under gentle agitation. The membranes were washed with $1 \%$ fat free milk solution in TBS-T (as above) and incubated with the secondary antibody (anti-human IgG peroxidase conjugated) (Sigma Co., St Louis, MO, USA) diluted in TBS-T at a 1:1000 ratio for two hours at $4{ }^{\circ} \mathrm{C}$ under gentle agitation. After washing with TBS, the membranes were incubated with the ECL Prime Western Blotting Detection Reagent (GE Healthcare, Menlo Park, CA, USA) according to the manufacturer's recommendations, and the antigenic spots were visualized using a Molecular Imaging ChemiDoc XRS system (Bio-Rad, Hercules, CA, USA).

\subsection{Protein Identification}

Spots of interest were manually excised from the preparative 2-DE gels. These spots were destained, shrunk, vacuum-dried, as described elsewhere [27] and then, were incubated with $12.5 \mathrm{ng} / \mu \mathrm{L}$ sequencing grade trypsin (Promega, Madison, WI, USA) overnight at $37{ }^{\circ} \mathrm{C}$. After digestion, the supernatants were separated and the peptides were extracted twice into $0.5 \%$ trifluoroacetic acid $/ 50 \%$ acetonitrile and once into $100 \%$ acetonitrile. These extracts were pooled, and their volumes were vacuum-dried. The derived concentrated peptide suspension for each spot of interest was spotted on a MALDI target plate, mixed with a saturated solution of matrix $\alpha$-cyano-4-hydroxytrans-cinnamic acid (Sigma Co., St Louis, MO, USA) and allowed to air-dry at room temperature. The samples were analyzed with a 5800 AB-SCIEX MALDI-TOF/TOF mass spectrometer (Applied Biosystems, Foster City, CA, USA) in automated mode. A MALDI MS spectrum was acquired from each spot (800 shots/spectrum), and 10 precursor peaks with a signal-to-noise ratio greater than 40 in at least two consecutive fractions were automatically selected for MS/MS analysis (4000 shots/spectrum). A collision energy of $1 \mathrm{keV}$ was used with air as the collision gas. All mass spectra were externally calibrated using the mass standards kit for the 4700 proteomics analyzer (Applied Biosystems, Foster City, CA, USA). The spectra were searched against an in-house database constructed using "A. fumigatus" as the selection criteria in Protein Pilot software using the Paragon algorithm (Applied Biosystems, Foster City, CA, USA). The name of the ORF (open reading frame) from A. fumigatus was found in the UniProt (Universal Protein Resource) server using the UniProt Knowledge/Swiss-Prot database.

\subsection{Homology Analysis}

The sequences of the antigenic proteins were aligned and compared using the protein BLAST tool of the NCBI database (http://blast.ncbi.nlm.nih.gov). The sequences of the identified A. fumigatus proteins were compared with sequences of human proteins and with proteins from other microorganisms. The selected microorganisms for comparison in the BLAST analyses are the etiological agents of mycosis that can be confused (diagnostically) with invasive aspergillosis (Rizophus spp. and other fungi of the Mucorales order, Penicillium spp., Paracoccidioides brasilienisis, Fusarium spp. and Paecilomyces spp.). The proteins with identity values lower than $40 \%$ and E-values higher than $1 \times 10^{-50}$ were identified to have no homology. 


\section{Conclusions}

Two antigenic proteins of $A$. fumigatus are described in this work as putative candidates for the immunodiagnostic of invasive aspergillosis: cytochrome P450 and eEF-3. These proteins presented no homology with human proteins and low homology with etiological agents of other IFIs. Among these, the elongation factor eEF-3 identified in A. fumigatus germlings is the most promising candidate once it shows the lowest homology with proteins of other fungal species that cause infections, which could be misdiagnosed with invasive aspergillosis.

\section{Acknowledgments}

This work was supported by Conselho Nacional de Desenvolvimento Científico e Tecnológico (CNPq) - grant 306582/2011-2, Fundação Carlos Chagas de Amparo à Pesquisa do Estado do Rio de Janeiro (FAPERJ) - grant E-26/102.819/2012 and Fundação de Amparo à Pesquisa do Estado de São Paulo(FAPESP) - grant 2012/50212-1. LMLB is a research fellow of CNPq and Faperj.

We would like to thank Aline Penna-de-Carvalho, technician of the Anatomy Department from Instituto de Biologia Roberto Alcântara Gomes for the help using the Molecular Imaging ChemiDoc XRS system (Bio-Rad) and Constancia Lima Diogo for the preparation of the bank of serum at Hospital 2.

\section{Author Contributions}

Emylli Dias Virginio had performed all experiments and data analysis.

Paula H. Kubitschek-Barreira contributed with the MS/MS data acquisition and protein identification analysis.

Marcelo R. Schirmer and Eliana Abdelhay contributed with all serum samples from Hospital 1 (proven and probable aspergillosis patients) and, are responsible for the clinical data of these groups of patients.

Maria Aparecida Shikanai-Yasuda and Marjorie V. Batista had collected and classified all serum samples from Hospital 2 (proven aspergillosis patients, patients with other mycoses and negative control group) and, are responsible for the clinical data of these groups of patients.

Leila M. Lopes-Bezerra is the intellectual mentor of this work and therefore, had contributed with the experimental design and revision of the manuscript.

\section{Conflicts of Interest}

The authors declare no conflict of interest.

\section{References}

1. Patterson, T.F. Risk stratification for invasive aspergillosis: Early assessment of host susceptibility. Med. Mycol. 2009, 47, S255-S260.

2. Steinbach, W.J.; Stevens, D.A.; Denning, D.W.; Moss, R.B. Advances against aspergillosis. Clin. Infect. Dis. 2003, 37, S155-S156.

3. Denning, D.W. Invasive aspergillosis. Clin. Infect Dis. 1998, 26, 781-805. 
4. Duthie, R.; Denning, D.W. Aspergillus fungemia: Report of two cases and review. Clin. Infect. Dis. 1995, 20, 598-605.

5. Ascioglu, S.; Rex, J.H.; de Pauw, B.; Bennett, J.E.; Bille, J.; Crokaert, F., Denning, D.W.; Donnelly, J.P.; Edwards, J.E.; Erjavec, Z.; et al. Invasive fungal infections cooperative group of the European Organization for research and treatment of cancer; mycoses study group of the National Institute of allergy and infectious diseases. Defining opportunistic invasive fungal infections in immunocompromised patients with cancer and hematopoietic stem cell transplants: An international consensus. Clin. Infect. Dis. 2002, 34, 7-14.

6. Soubani, A.O.; Khanchandani, G.; Ahmed, H.P. Clinical significance of lower respiratory tract Aspergillus culture in elderly hospitalized patients. Eur. J. Clin. Microbiol. Infect. Dis. 2004, 23, 491-494.

7. Latgé, J.P. Aspergillus fumigatus and Aspergillosis. Clin. Microbiol. Rev. 1999, 12, 310-350.

8. Thornton, C.R. Detection of invasive Aspergillosis. Adv. Appl. Microbiol. 2010, 70, 187-216.

9. Mennink-Kersten, M.A.; Donnelly, J.P.; Verweij, P.E. Detection of circulating galactomannan for the diagnosis and management of invasive aspergillosis. Lancet Infect. Dis. 2004, 4, 349-357.

10. Chamilos, G.; Kontoyiannis, D.P. Defining the diagnosis of invasive aspergillosis. Med. Mycol. 2006, 44, 163-172.

11. Beauvais, A.; Schmidt, C.; Guadagnini, S.; Roux, P.; Perret, E.; Henry, C.; Paris, S.; Mallet, A.; Prévost, M.C.; Latgé, J.P. An extracellular matrix glues together the aerial-grown hyphae of Aspergillus fumigatus. Cell Microbiol. 2007, 9, 1588-1600.

12. Fontaine, T.; Simenel, C.; Dubreucq, G.; Adam, O.; Delepierre, M. Molecular organization of the alkali-insoluble fraction of Aspergillus fumigatus cell wall. J. Biol. Chem. 2000, 275, 27594-27607.

13. Xavier, M.O.; Pasqualotto, A.C.; Cardoso, I.C.; Severo, L.C. Cross-reactivity of Paracoccidioides brasiliensis, Histoplasma capsulatum, and Cryptococcus species in the commercial Platelia Aspergillus enzyme immunoassay. Clin. Vaccine Immunol. 2009, 16, 132-133.

14. Adam, O.; Aupérin, A.; Wilquin, F.; Bourhis, J.H.; Gachot, B.; Chachaty, E. Treatment with piperacillin-tazobactam and false-positive Aspergillus galactomannan antigen test results for patients with hematological malignancies. Clin. Infect. Dis. 2004, 38, 917-920.

15. Mikulska, M.; Furfaro, E.; del Bono, V.; Raiola, A.M.; Ratto, S.; Bacigalupo, A.; Viscoli, C. Piperacillin/tazobactam $\left(\right.$ Tazocin $^{\mathrm{TM}}$ ) seems to be no longer responsible for false-positive results of the galactomannan assay. J. Antimicrob. Chemother. 2012, 67, 1746-1748.

16. Tortorano, A.M.; Esposto, M.C.; Prigitano, A.; Grancini, A.; Ossi, C.; Cavanna, C.; Cascio G.L. Cross-reactivity of Fusarium spp. in the Aspergillus Galactomannan enzyme-linked immunosorbent assay. J. Clin. Microbiol. 2012, 50, 1051-1053.

17. Wheat, L.J.; Hackett, E.; Durkin, M.; Connolly, P.; Petraitiene, R.; Walsh, T.J.; Knox, K.; Hage, C. Histoplasmosis-associated cross-reactivity in the BioRad Platelia Aspergillus enzyme immunoassay. Clin. Vaccine Immunol. 2007, 14, 638-640. 
18. De Pauw, B.; Walsh, T.J.; Donnelly, J.P.; Stevens, D.A.; Edwards, J.E.; Calandra, T.; Pappas, P.G.; Maertens, J.; Lortholary, O.; Kauffman, C.A.; et al. Revised definitions of invasive fungal disease from the European Organization for research and treatment of cancer/invasive fungal infections cooperative group and the National Institute of allergy and infectious diseases mycoses study group (EORTC/MSG) consensus group. Clin. Infect. Dis. 2008, 46, 1813-1821.

19. Kniemeyer, O.; Lessing, F.; Brakhage, A.A. Proteome analysis for pathogenicity and new diagnostic markers for Aspergillus fumigatus. Med. Mycol. 2009, 47, S248-S254.

20. Singh, B.; Oellerich, M.; Kumar, R.; Kumar, M.; Bhadoria, D.P.; Reichard, U.; Gupta, V.K.; Sharma, G.L.; Asif, A.R. Immuno-reactive molecules identified from the secreted proteome of Aspergillus fumigatus. J. Proteome Res. 2010, 9, 5517-5529.

21. Singh, B.; Sharma, G.L.; Oellerich, M.; Kumar, R.; Singh, S.; Bhadoria, D.P.; Katyal, A.; Reichard, U.; Asif, A.R. Novel cytosolic allergens of Aspergillus fumigatus identified from germinating conidia. J. Proteome Res. 2010, 9, 5530-5541.

22. Kumar, A.; Ahmed, R.; Singh, P.K.; Shukla, P.K. Identification of virulence factors and diagnostic markers using immunosecretome of Aspergillus fumigatus. J. Proteomics 2011, 74, 1104-1112.

23. Asif, A.R.; Oellerich, M.; Amstrong, V.W.; Gross, U.; Reichard. U. Analysis of the cellular Aspergillus fumigatus proteome that reacts with sera from rabbits developing an acquired immunity after experimental aspergillosis. Electrophoresis 2010, 31, 1947-1958.

24. Shi, L.N.; Li, F.Q.; Huang, M.; Lu, J.F.; Kong, X.X.; Wang, S.Q.; Shao, H.F. Immunoproteomics based identification of thioredoxin reductase GliT and novel Aspergillus fumigatus antigens for serologic diagnosis of invasive aspergillosis. BMC Microbiol. 2012, 12, 11.

25. Sipsas, N.V.; Kontoyiannis, D.P. Invasive fungal infections in patients with cancer in the Intensive Care Unit. Int. J. Antimicrob. Agents 2012, 39, 464-471.

26. Denning, D.W. Aspergillus species. In Principles and Practice of Infectious Diseases, 5th ed.; Mandell, G.L., Bennett, J.E., Dolin, R., Eds.; Churchill Livingstone: Philadelphia, PA, USA, 2000; Volume 2, pp. 2674-2685.

27. Pitarch, A.; Jiménez, A.; Nombela, C.; Gil, C. Decoding serological response to Candida cell wall immunome into novel diagnostic, prognostic, and therapeutic candidates for systemic candidiasis by proteomic and bioinformatic analyses. Mol. Cell. Proteomics 2006, 5, 79-96.

28. Lai, H.Y.; Tam, M.F.; Tang, R.B.; Chou, H.; Chang, C.Y.; Tsai, J.J.; Shen, H.D. cDNA cloning and immunological characterization of a newly identified enolase allergen from Penicillium citrinum and Aspergillus fumigatus. Int. Arch. Allergy Immunol. 2002, 127, 181-190.

29. Rohde, M.; Schwienbacher, M.; Nikolaus, T.; Heesemann, J.; Ebel, F. Detection of early phase specific surface appendages during germination of Aspergillus fumigatus conidia. FEMS Microbiol. Lett. 2002, 206, 99-105.

30. Rhodes, C.J. Aspergillus fumigatus: Growth and virulence. Med. Mycol. 2006, 44, 77-81.

31. Kubitschek-Barreira, P.H.; Curty, N.; Neves, G.W.; Gil, C.; Lopes-Bezerra, L.M. Differential proteomic analysis of Aspergillus fumigatus morphotypes reveals putative drug targets. J. Proteomics 2013, 78, 522-534.

32. Denikus, N.; Orfaniotou, F.; Wulf, G.; Lehmann, P.F.; Monod, M.; Reichard, U. Fungal antigens expressed during invasive aspergillosis. Infect. Immun. 2005, 73, 4704-4713. 
33. Lamaris, G.A.; Chamilos, G.; Lewis, R.E.; Safdar, A.; Raad, II.; Kontoyiannis, D.P. Scedosporium infection in a tertiary care cancer center: A review of 25 cases from 1989-2006. Clin. Infect. Dis. 2006, 43, 1580-1584.

34. Cortez, K.J.; Roilides, E.; Quiroz-Telles, F.; Meletiadis, J.; Antachopoulos, C.; Knudsen, T.; Buchanan, W.; Milanovich, J.; Sutton, D.A.; Fothergill, A.; et al. Infections caused by Scedosporium spp. Clin. Microbiol. Rev. 2008, 21, 157-197.

35. Mori, Y.; Nagasaki, Y.; Kamezaki, K.; Takenaka, K.; Iwasaki, H.; Harada, N.; Miyamoto, T.; Abe, Y.; Shimono, N.; Akashi, K.; et al. High incidence of false-positive Aspergillus galactomannan test in multiple myeloma. Am. J. Hematol. 2010, 5, 449-451.

36. Morrissey, C.O. Advancing the field: Evidence for new management strategies in invasive fungal infections. Curr. Fungal. Infect. Rep. 2013, 7, 51-58.

37. Vidovic, A.; Arsic-Arsenijevic, V.; Tomin, D.; Djunic, I.; Jakovic, R.; Loncar, Z.; Barac, A. Proven invasive pulmonary mucormycosis successfully treated with amphotericin B and surgery in patient with acute myeloblastic leukemia: A case report. J. Med. Case Rep. 2013, 7, 263.

38. Crešnar, B.; Petrič, S. Cytochrome P450 enzymes in the fungal kingdom. Biochim. Biophys. Acta 2010, 1814, 29-35.

39. Ehrlich, K.C.; Chang, P.K.; Yu, J.; Cotty, P.J. Aflatoxin biosynthesis cluster gene cypA is required for $\mathrm{G}$ aflatoxin formation. Appl. Environ. Microbiol. 2004, 70, 6518-6524.

40. Wen, Y.; Hatabayashi, H.; Arai, H.; Kitamoto, H.K.; Yabe, K. Function of the cypX and moxY genes in aflatoxin biosynthesis in Aspergillus parasiticus. Appl. Environ. Microbiol. 2005, 71, 3192-3198.

41. Lepesheva, G.I.; Waterman, M.R. Sterol 14- $\alpha$-demethylase cytochrome P450 (CYP51), a P450 in all biological kingdoms. Biochim. Biophys. Acta 2007, 1770, 467-477.

42. Verweij, P.E.; Mellado, E.; Melchers, W. Multiple-triazole-resistant aspergillosis. N. Engl. J. Med. 2007, 356, 1481-1483.

43. Mavridou, E.; Bruggemann, R.J.; Melchers, W.J.; Verweij, P.E.; Mouton, J.W. Impact of cyp51A mutations on the pharmacokinetic and pharmacodynamics properties of voriconazole in a murine model of disseminated aspergillosis. Antimicrob. Agents Chemother. 2010, 54, 4758-4764.

44. Kuipers, S.; Brüggemann, R.J.; de Sévaux, R.G.; Heesakkers, J.P.; Melchers, W.J.; Mouton, J.W.; Verweij, P.E. Failure of posaconazole therapy in a renal transplant patient with invasive aspergillosis due to Aspergillus fumigatus with attenuated susceptibility to posaconazole. Antimicrob. Agents Chemother. 2011, 55, 3564-3566.

45. Van den Brink, H.M.; van Gorcom, R.F.; van den Hondel, C.A.; Punt, P.J. Cytochrome P450 enzyme systems in fungi. Fungal Genet. Biol. 1998, 23, 1-17.

46. Nelson, D.R. Cytochrome P450 and the individuality of species. Arch. Biochem. Biophys. 1999, 369, 1-10.

47. Nelson, D.R. Cytochrome P450 nomenclature. Methods Mol. Cell. Biol. 1998, 107, 15-24.

48. Nelson, D.R. Metazoan cytochrome P450 evolution. Comp. Biochem. Physiol. C Pharmacol. Toxicol. Endocrinol. 1998, 121, 15-22.

49. Hinnebusch, A.G.; Dever, T.E.; Asano, K. Translational Control in Biology and Medicine; Sonenberg, N., Hershey, J.W.B., Mathews, M.B., Eds.; Cold Spring Harbor Laboratory Press: Cold Spring Harbor, NY, USA, 2007; pp. 225-268. 
50. Carvalho, M.D.; Carvalho, J.F.; Merrick, W.C. Biological characterization of various forms of elongation factor 1 from rabbit reticulocytes. Arch. Biochem. Biophys. 1984, 234, 603-611.

51. Skogerson, L.; Moldave, K. Characterization of the interaction of aminoacyltransferase II with ribosomes. Binding of transferase II and translocation of peptidyl transfer ribonucleic acid. J. Biol. Chem. 1968, 243, 5354-5360.

52. Liu, M.; Gelli, A. Elongation factor 3, EF3, associates with the calcium channel Cch1 and targets Cch1 to the plasma membrane in Cryptococcus neoformans. Eukaryot. Cell 2008, 7, 1118-1126.

53. Hollenstein, K.; Dawson, R.J.; Locher, K.P. Structure and mechanism of ABC transporter proteins. Curr. Opin. Struct. Biol. 2007, 17, 412-418.

54. Uritani, M.; Miyazaki, M. Characterization of the ATPase and GTPase activities of the elongation factor 3 (EF-3) from yeasts. J. Biochem. 1988, 103, 522-530.

55. Sasikumar, A.N.; Kinzy, T.G. Mutations in the chromodomain-like insertion of translation elongation factor 3 compromise protein synthesis through reduced ATPase activity. J. Biol. Chem. 2014, 289, 4853-4860.

56. López-Ribot, J.L.; Alloush, H.M.; Masten, B.J.; Chaffin, W.L. Evidence for presence in the cell wall of Candida albicans of a protein related to the hsp70 family. Infect. Immun. 1996, 64, 3333-3340.

57. Yuan, S.Y. Protein kinase signalling in the modulation of microvascular permeability. Vascul. Pharmacol. 2002, 39, 213-223.

58. Pardo, M.; Ward, M.; Bains, S.; Molina, M.; Blackstock, W.; Gil, C.; Nombela C. A proteomic approach for the study of Saccharomyces cerevisiae cell wall biogenesis. Electrophoresis 2000, 21, 3396-3410.

59. Pitarch, A.; Sanchez, M.; Nombela, C.; Gil, C. Sequential fractionation and two-dimensional gel analysis unravels the complexity of the dimorphic fungus Candida albicans cell wall proteome. Mol. Cell. Proteomics 2002, 1, 967-982.

(C) 2014 by the authors; licensee MDPI, Basel, Switzerland. This article is an open access article distributed under the terms and conditions of the Creative Commons Attribution license (http://creativecommons.org/licenses/by/3.0/). 